Boletín de la Sociedad Geológica Mexicana

Volumen 65, NÚM. 1, 2013, P. 83-98

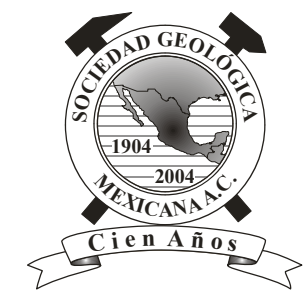

\title{
Caracterización y ambiente tectónico de las rocas máficas del Complejo El Chilar: evidencias de un prisma de acreción pre-Jurásico Tardío en el centro de México
}

\author{
Víctor M. Dávila-Alcocer ${ }^{1, *}$, Elena Centeno-García ${ }^{1}$, Lorenzo Meriggi ${ }^{2}$ \\ ${ }^{1}$ Departamento de Geología Regional, Instituto de Geología, Universidad Nacional Autónoma de México. Ciudad Universitaria, \\ 04510, México D.F., México \\ ${ }^{2}$ MASSA (Metodologie e Applicazioni Scientifiche per la Salvaguardia Ambientale) spin-off S.r.l., Largo Guido Novello 1/C 50126 \\ Florencia, Italia \\ *davilal@unam.mx
}

\begin{abstract}
Resumen
El Complejo El Chilar aflora en el estado de Querétaro y es una unidad sedimentaria muy deformada a tal grado que forma una mezcla tectónica (mélange). Este complejo de edad pre-Jurásico Tardío, contiene bloques autóctonos de arenisca o arenisca con intercalaciones de lutita así como bloques exóticos de pedernal con horizontes de radiolaritas. El bloque exótico de mayor tamaño tiene $1.5 \mathrm{~km}$ de longitud y aflora en las inmediaciones del poblado El Terrero. Este bloque está cortado por diques de microgabro, de los cuales se analizaron 13 muestras con el fin de caracterizar su ambiente tectónico. Se hicieron análisis petrográficos, de fluorescencia de rayos-X (FRX), de espectrometría de masas por inducción acoplada de plasma (ICPMS), de difracción de rayos-X (DRX) y con microsonda electrónica. Los análisis de roca total de elementos mayores y traza, incluyendo el análisis de tierras raras, nos han permitido caracterizar dos grupos de muestras. El grupo I está formado por microgabro con clinopiroxeno abundante, albita y algo de mica. El clinopiroxeno muestra bordes de anfíbol, mientras que la albita está parcialmente reemplazada por hialofano, siendo este último mineral y la mica los portadores de Ba en cantidades tan grandes como $9000 \mathrm{ppm}$. El grupo II está formado por microgabro rico en feldespato sódico con relictos de clinopiroxeno.

Todas las muestras estudiadas presentan firmas geoquímicas típicas de una fuente de manto enriquecida (de tipo basaltos de isla oceánica), similares a las que presentan algunas provincias actuales del Pacífico de tipo basaltos de isla oceánica. La composición de los diques de microgabro es igual a la de algunos basaltos del estado de Zacatecas contenidos también en bloques exóticos en un prisma de acreción similar. Con base en esta y otras similitudes se establece una probable distribución regional del prisma de acreción.

Consideramos que fluidos de subducción a profundidades someras alteraron a los diques de microgabro durante la acreción, originando enriquecimiento en elementos litófilos de radio iónico grande (principalmente Ba y K).
\end{abstract}

Palabras clave: Mélange, Complejo El Chilar, geoquímica, basaltos de isla oceánica, acreción, Querétaro.

\begin{abstract}
The El Chilar Complex, located in Queretaro State, is a strongly deformed sedimentary unit that forms a tectonic mélange. This complex contains autochthonous blocks of sandstone or sandstone interbedded with shale, as well as exotic blocks of radiolarian chert. It is pre-Jurassic in age, and contains sandstone or sandstone with interbedded shale autocthonous blocks as well as chert exotic blocks. The largest of the exotic blocks is $1.5 \mathrm{~km}$. long, and crops out in the town of El Terrero. This chert block is intruded by microgabbro dikes, from which 13 samples were analyzed. Petrography, XRF, ICPMS, XRD and microprobe analyses were done in order to characterize the tectonic setting of the mafic rocks. Whole rock major and trace element analysis, including rare earth element analysis, allowed us to characterize two groups of samples. Group I comprises microgabbro with abundant clinopyroxene and albite and scarce mica.
\end{abstract}


Clinopyroxene shows rims of amphibole, and albite is partially replaced by hyalophane. Group I is enriched in Ba (9000 ppm) which is concentrated in micas and hyalophane. Group II is formed by Na feldspar-rich microgabbro with few relicts of clinopyroxene.

All samples show geochemical signatures of typical enriched mantle source (oceanic island basalts), similar to those reported from recent oceanic island basalts on the Pacific Ocean floor. Microgabbro dikes described in this paper have geochemical signatures similar to those of mafic rocks that form exotic blocks within an accretionary prism in northern Zacatecas State. This and other similarities suggest that both localities belong to the same accretionary prism of regional extent. Our results show that the exotic block of El Terrero contains dikes of geochemical affinity,

We consider that shallow subduction fluids altered the microgabbro dikes during accretion, originating large ion lithophile elements enrichment (mainly Ba and K).

Keywords: Mélange, El Chilar Complex, geochemistry, ocean island basalts, accretion, Querétaro.

\section{Introducción}

El Complejo El Chilar es una unidad sedimentaria fuertemente cizallada que presenta zonas localizadas, con milonitización y/o metamorfismo de bajo grado. Las rocas de este complejo afloran en la parte central del estado de Querétaro, al sureste del poblado de San Pablo Tolimán (Figura 1), y a 250 kilómetros al norte de la ciudad de México. Anteriormente, estas rocas fueron agrupadas con rocas volcanoclásticas del Jurásico Superior en una sola unidad, denominada formación San Juan de la Rosa (Carrillo-Martínez, 1998). Sin embargo, la cartografía detallada de la región permitió identificar una discordancia importante entre la secuencia sedimentaria más antigua con fuerte deformación y la sucesión volcanoclástica que pertenece a la formación San Juan de la Rosa (DávilaAlcocer et al., 2009). Esta unidad sedimentaria deformada fue nombrada de manera informal como formación El Chilar por López-Ramos (1985).

Recientemente, la formación El Chilar, fue denominada Complejo El Chilar por Dávila-Alcocer et al. (2009), cuyo contacto superior es una discordancia angular con la formación San Juan de la Rosa, misma que cambia gradualmente a la sucesión calcárea del Cretácico (formaciones Tamaulipas y Soyatal), (Figura 1B) y que a su vez son cubiertas por varias unidades volcánicas del Cenozoico, así como por depósitos aluviales y fluviales del Cuaternario (Carrillo-Martínez, 1998). La misma columna estratigráfica, solo se vuelve a encontrar en los alrededores del domo volcánico de Peña de Bernal, localizado a $20 \mathrm{~km}$ al suroeste de San Pablo Tolimán, en ninguna otra región circundante se han identificado afloramientos del Complejo El Chilar.

El presente artículo se enfoca a la interpretación petrotectónica de los resultados petrográficos y geoquímicos de las escasas rocas ígneas que contiene el Complejo El Chilar y que son de suma importancia para caracterizar el ambiente tectónico de dicho complejo.

\section{Geología del Complejo El Chilar}

La unidad que López-Ramos (1985) describe informalmente bajo el nombre de formación El Chilar, es en realidad un cuerpo de roca que ha perdido su estratigrafía original y estructura primaria por tectonismo y por lo tanto no obedece a la Ley de Superposición de los estratos (ver Código Estratigráfico Norteamericano, 2005). Su estructura corresponde a lo que en la literatura se menciona como una fábrica de bloques contenidos en matriz (Kusky y Bradley, 1999; Medley, 2002) y que está presente en algunos mélanges tectónicos (Festa et al., 2010; Berkland et al., 1972).

En el Complejo El Chilar se presentan bloques que tienen dimensiones muy variadas que van desde cientos de metros a escasos centímetros y que flotan en una matriz areno-limosa, en su mayoría presentan formas sigmoidales que son producto de la intensa deformación por cizalla (Figura 2a). Por lo anterior, se considera como una unidad litodémica a la cual se le ha denominado Complejo El Chilar (Dávila-Alcocer y Centeno-García, 2006), que de acuerdo al Código Estratigráfico Norteamericano (NACSN, 2005, art. 37) puede ser clasificado como un complejo estructural.

Este complejo es de gran importancia ya que constituye la unidad más antigua de la región. Litológicamente, está formado por gruesos paquetes de arenisca, algunas veces con algo de lutita, e intervalos con alternancia de arenisca y lutita. También se encuentran escasos conglomerados polimícticos (rellenos de canal y flujos de detritos). Dichas litologías tienen una composición rica en clastos de cuarzo. En las zonas menos deformadas se observan columnas de pocos metros de espesor con una estratigrafía coherente, donde se han preservado estructuras primarias, las cuales sugieren que el depósito se dio en forma de flujos turbidíticos.

La pérdida del orden estratigráfico de una sucesión como resultado de un proceso continuo de deformación sugiere una cizalla muy intensa, similar a aquella observada en zonas de fallas mayores a nivel cortical, o bien en prismas de acreción formados en zonas de subducción, lo que conlleva 


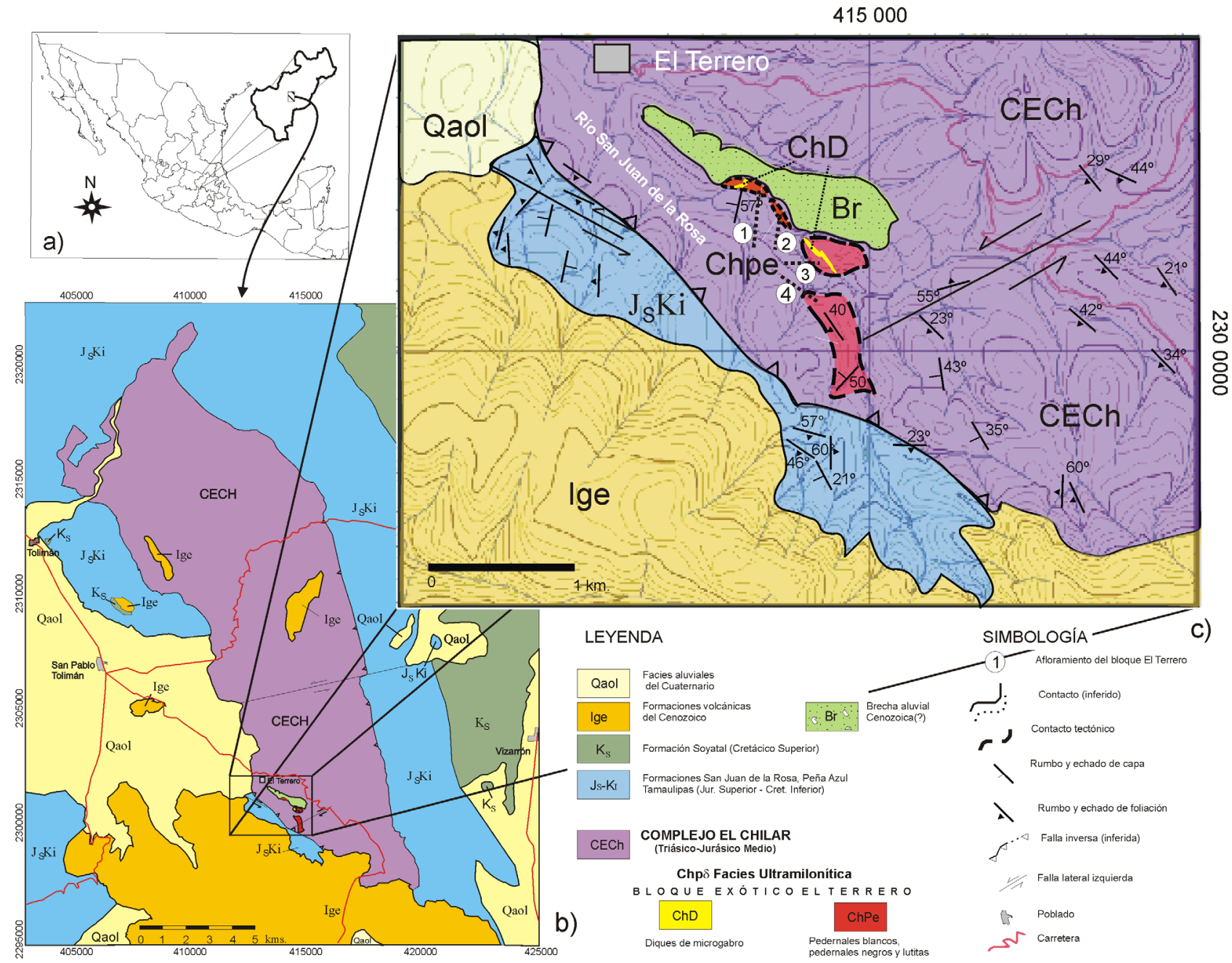

Figura 1. a) Mapa de ubicación del área de estudio. b) Mapa geológico generalizado de la región de Tolimán. c) Ampliación de la zona donde aflora el bloque exótico El Terrero mostrando los afloramientos 1 y 3 de microgabro discutidos en el presente trabajo.

a variaciones regionales en la deformación. Para facilitar su descripción Raymond (1984) distingue cuatro tipos de unidades que denomina con las letras griegas $\alpha, \beta, \gamma, \delta$ siendo esta última la de mayor intensidad en la deformación, caracterizada por la presencia de bloques exóticos y una fábrica de bloques dentro de una matriz y a la cual aplica el término mélange.

Las características estructurales del Complejo El Chilar son las de un mélange tectónico, constituido por bloques tanto autóctonos como alóctonos envueltos en la matriz areno-pelítica rica en cuarzo. La cuarzoarenita es el principal constituyente de los bloques autóctonos, mientras que el pedernal con horizontes de radiolaritas, algunas veces interestratificados con lutitas, conforman los bloques exóticos. Estos últimos se consideran como "bloques exóticos" porque los pedernales no se han observado en relación sedimentaria (interestratificados) con la matriz que los envuelve. Las características de los pedernales y la presencia de radiolaritas sugieren que estos probablemente formaban parte de los niveles estratigráficos superiores de una placa oceánica.

Dentro del Complejo El Chilar, las rocas con mayor deformación están expuestas en las riveras del río San Juan de la Rosa, a la altura del poblado El Terrero, y corresponden a afloramientos de rocas que van de brecha y milonita a gruesas bandas de ultramilonita que aparecen localmente (Figura $1 \mathrm{C}, \mathrm{Chp \delta}$ ). En dicha zona, y en otras zonas de cizalla, el Complejo El Chilar está constituido por bloques con formas sigmoidales cuya litología es variable, presentando consistentemente zonas de cizalla en sus límites con la matriz que los contiene. Esta última está intensamente deformada y como se mencionó anteriormente, se compone de rocas sedimentarias clásticas ricas en cuarzo. Los bloques encontrados se clasifican en: a) bloques autóctonos formados por intercalaciones de cuazoarenita y limolita cuarcítica, algunas veces llega a estar presente algo de lutita cuya composición es similar a la de la matriz; y b) bloques alóctonos o exóticos que ocurren en una asociación litológica ajena a la matriz que los contiene y cuyas litologías nunca muestran relaciones 


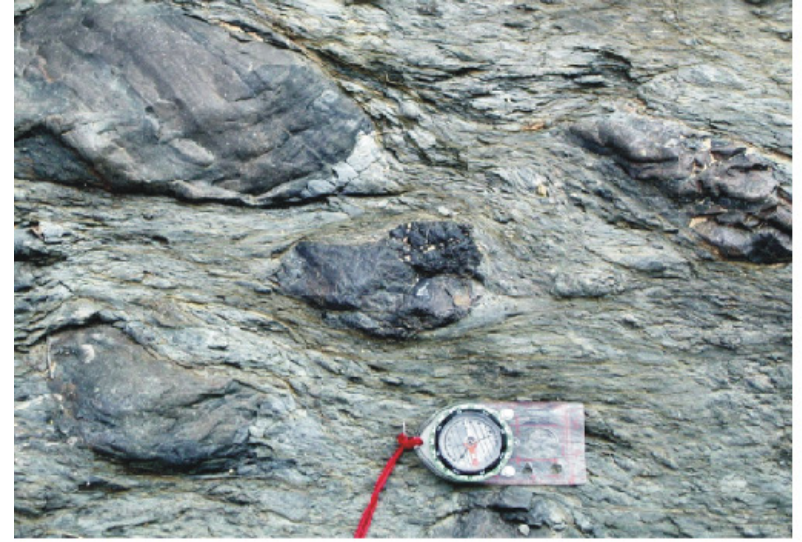

a)

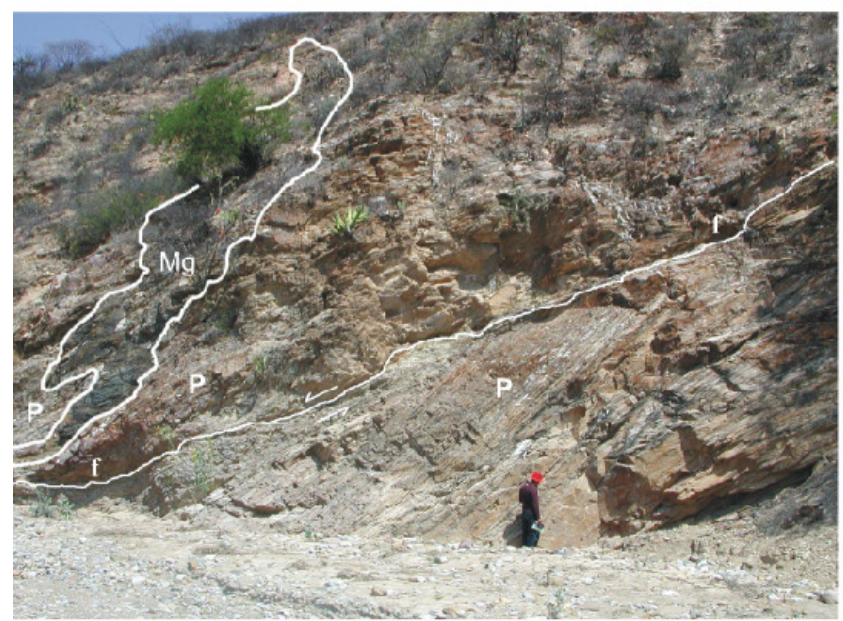

c)

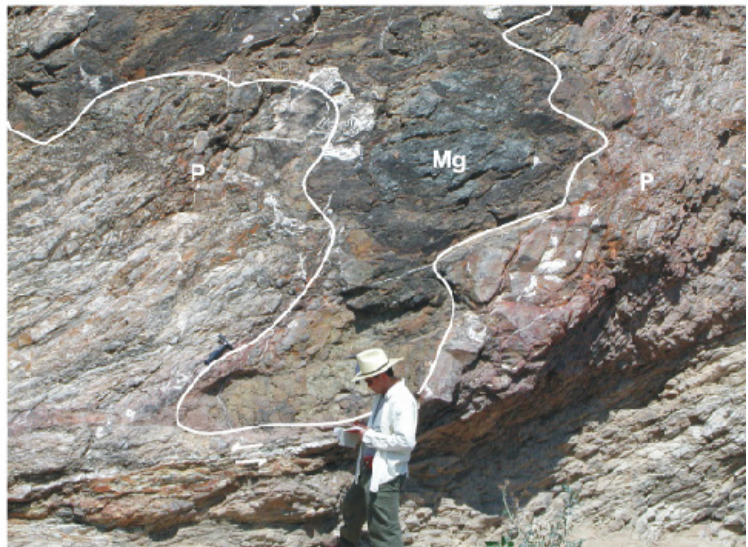

b)

Figura 2. a) Bloques de pedernal (centro) y arenisca con formas sigmoidales en una matriz clástica intensamente cizallada (zona de milonita a ultramilonita). b) Detalle del afloramiento de la facies de pedernales blancos impuros $(\mathrm{P})$ donde se muestra la relación cortante del dique de microgabro $(\mathrm{Mg})$.c) Panorámica del afloramiento principal del bloque El Terrero con el dique de microgabro delineado (Mg) cortando la secuencia de pedernales (P). d) Afloramiento de pedernales negros con radiolaritas intercalados con lutitas que constituyen a uno de los afloramientos del bloque tectónico.

de depósito o estratigráficas con las litologías que forman la matriz (Figura 2a). La naturaleza de estos últimos es de gran importancia en nuestro trabajo, en particular aquellos que están cortados por diques de rocas máficas, ya que la caracterización de dichas rocas ígneas puede aportar las bases para establecer su entorno tectónico.

El bloque exótico con mayor dimensión y significado encontrado dentro del Complejo El Chilar es aquel localizado en las cercanías de El Terrero de donde toma su nombre (bloque El Terrero), con $1.5 \mathrm{~km}$ de longitud y 500 $\mathrm{m}$ de ancho. El bloque exótico El Terrero es disectado por el río San Juan de la Rosa y alguno de sus afluentes dejándolo expuesto en cuatro afloramientos (Figura 1C, Chpe, 1, 2, $3,4)$. El bloque está formado por pedernales pelágicos con horizontes de radiolaritas e intercalaciones de limos y lutitas. En dos de estos afloramientos (1 y 3), los pedernales son cortados por diques de rocas ígneas (Figura $1 \mathrm{C}, \mathrm{ChD}$;
Figuras $2 \mathrm{~b}$, y $2 \mathrm{c}$ ). Por su relación de campo, textura relativamente gruesa y color oscuro, preliminarmente se consideró a estos diques como máficos y de naturaleza hipabisal. A la fecha no se han encontrado afectando a otros bloques o a la matriz del Complejo El Chilar.

A detalle, en dichos afloramientos se pueden distinguir tres tipos de litologías: a) pedernal blanco impuro que predomina en los afloramientos Chpe1, 2, 3; b) pedernal negro formado por cuarzo criptocristalino en el que se observa el desarrollo de cristales de sericita con orientación preferencial. Algunos de estos pedernales contienen abundantes radiolarios $(>60 \%)$ constituyendo así verdaderos horizontes de radiolaritas. Sin embargo, los radiolarios están mal conservados así como cuerpos reniformes de posibles moldes internos de Raxellas(?). Se presentan en estratos delgados interestratificados con horizontes también delgados de lutita (Figura 2d) y son 
la litología predominante en el afloramiento 4. La edad de las radiolaritas aún no está determinada por la mala preservación de esta microfauna; c) diques máficos cortando a las dos litologías anteriores alcanzando hasta 2 metros de ancho y algunas ramificaciones más delgadas (Figuras $2 \mathrm{~b}$ y 2c). Estos diques se encuentran afectados por la misma deformación y cizallamiento que presentan los pedernales en los que se emplazan.

El núcleo del bloque El Terrero contrasta por su menor deformación, en comparación con su borde exterior y la matriz que lo rodea, pudiendo distinguirse en él estratos delgados a medianos plegados, en parte afectados por zonas de cizalla de menor escala (Figura 2).

Los afloramientos 1, 2, 3 están parcialmente cubiertos por una brecha de origen aluvial (br) más reciente, (Figura 1C) y de carácter aparentemente local, por lo que no ha sido asociada a ninguna unidad.

Por su posición estratigráfica se considera que la edad del Complejo El Chilar es pre-Jurásico tardío, ya que subyace de manera discordante a las rocas volcanoclásticas de la formación San Juan de la Rosa, la cual se considera de edad Jurásico Tardío-Cretácico Temprano (Dávila-Alcocer et al., 2009). Por otra parte, el depósito de las rocas sedimentarias del Complejo El Chilar no puede ser anterior al Pérmico, como lo sugiere el predominio de edades U-Pb tan jóvenes como $279 \mathrm{Ma}$, obtenidas de zircones detríticos de una muestra de arenisca (Dávila-Alcocer et al., 2008).

Turbiditas siliciclásticas litológicamente similares y con poblaciones de circones detríticos iguales a las obtenidas de la muestra de El Chilar (Dávila-Alcocer et al., 2008), afloran en varias localidades del centro del país, tales como la Formación Zacatecas en varias localidades del noreste de México (Barboza-Gudiño et al., 2010; Barboza-Gudiño, 2012), las formaciones La Ballena y Taray (Centeno-García y Silva-Romo, 1997; Silva-Romo et al., 2000, y DíazSalgado, 2004), y el Complejo Arteaga del occidente de México (Centeno-García et al., 1993). De éstas unidades, la formación Zacatecas en la Ciudad del mismo nombre, la formación Taray y el Complejo Arteaga presentan texturas de mélange tectónico, con bloques exóticos de pedernal y rocas máficas (Centeno-García et al., 1993, Centeno-García y Silva-Romo, 1997; Silva-Romo et al., 2000, y DíazSalgado, 2004, y Anderson et al., 2005). Por otra parte, algunas de las rocas sedimentarias antes mencionadas, con las que se hace una correlación por procedencia, contienen fósiles del Triásico, lo que sugiere que la matriz sedimentaria del Complejo El Chilar bien pudo depositarse durante el Triásico Tardío (Dávila-Alcocer et al., 2008).

\section{Metodología}

Se analizaron 13 muestras de los diques máficos que cortan al bloque El Terrero, once de ellas provienen del afloramiento 1 y las muestras $5 \mathrm{~A}$ y $5 \mathrm{~B}$ provienen del aflormaiento 3. Secciones delgadas de estas fueron analizadas petrográficamente. En secciones delgadas pulidas, se determinó con la microsonda JEOL-JXA8900/R del Laboratorio Universitario de Petrología (LUP) la composición mineral de clinopiroxenos, feldespatos potásicos y micas. Sucesivamente el estudio de microsonda permitió la determinación de las fases mineralógicas presentes y de las relaciones entre estas. La caracterización de los minerales se complemento con observaciones y fotografías con catodoluminicencia.

Todas las muestras se analizaron por óxidos mayores y elementos traza en los laboratorios del Instituto de Geología de la Universidad Nacional Autónoma de México. Para el análisis de los elementos mayores el equipo utilizado fue un espectrómetro secuencial de fluorescencia de rayos $\mathrm{X}$ Siemens SRS 3000. La pérdida por calcinación (PxC) se determino en $1 \mathrm{~g}$ de muestra seca, calentada a $1000^{\circ} \mathrm{C}$ por una hora. Para el análisis de elementos traza, el equipo utilizado fue un espectrómetro de masas con plasma inductivamente acoplado como fuente de ionización Agilent 7500 ce con celda de colisión (ICPMS Agilent 7500 ce). Además, se obtuvieron duplicados de elementos traza de las muestras 11B y 14 en los laboratorios Actlabs (Activation Laboratories) de Canadá por el método MS-FUS, de los cuales no se obtuvieron diferencias significativas en la mayoría de los elementos analizados por los laboratorios del Instituto de Geología. La abundancia de Ba en algunas muestras parece haber producido interferencia analítica con respecto al Eu. Las concentraciones de este elemento son inusualmente altas, razón por la cual se omitieron las concentraciones de Eu anómalas. En las Tablas 1 y 2 se reportan los datos geoquímicos obtenidos.

\section{Resultados}

\subsection{Petrografía}

Con base en los resultados de los estudios petrográficos, las muestras corresponden a microgabros y se pueden separar en dos grupos de acuerdo a sus diferencias mineralógicas y texturales:

\subsubsection{Grupo I}

Está compuesto por 9 muestras de la serie $0702(7,8$, $9,10,11 \mathrm{~A}, 11 \mathrm{~B}, 11 \mathrm{C}, 12,13,15)$ que petrográficamente exhiben una textura heterogranular, con fenocristales de clinopiroxeno elongados, euedrales y subedrales (Figura 3a, b), y que llegan a presentar un pleocroismo de violeta a incoloro, característico de la titano-augita. Estos fenocristales están rodeados por fases mineralógicas difíciles de reconocer pero que por DRX se caracterizaron como minerales arcillosos del tipo caolinita y trazas de posible clorita.

Las muestras 9, 11A, 11B de la serie 0702 son las menos alteradas, como lo muestra su menor valor de PxC (Tabla 1) y son la base para la caracterización petrográfica y por 
Tabla 1. Concentración de elementos mayores por FRX (Lab. UNAM) de trece muestras de los diques de microgabros del bloque Exótico El Terrero presente dentro del Complejo El Chilar.

\begin{tabular}{|c|c|c|c|c|c|c|c|c|c|c|c|c|c|}
\hline Muestra & 0702-5A & 0702-5B & 0702-7 & 0702-8 & 0702-9 & 0702-10 & 0702-11A & 0702-11B & 0702-11C & 0702-12 & 0702-13 & 0702-14 & 0702-15 \\
\hline $\begin{array}{l}\text { Longitud E } \\
\text { (UTM) }\end{array}$ & 414446 & 414446 & 414509.4 & 414509.4 & 414509.4 & 414509.4 & 414509.4 & 414509.4 & 414509.4 & 414218.1 & 414173.2 & 414138.5 & 414173.2 \\
\hline $\begin{array}{l}\text { Latitud N } \\
\text { (UTM) }\end{array}$ & $\begin{array}{l}2300679.5 \\
\text { (\% en peso) }\end{array}$ & $\begin{array}{l}2300679.5 \\
\text { (\% en peso) }\end{array}$ & $\begin{array}{c}2300694 \\
\text { (\% en peso) }\end{array}$ & $\begin{array}{c}2300694 \\
\text { (\% en peso) }\end{array}$ & $\begin{array}{c}2300694 \\
\text { (\% en peso) }\end{array}$ & $\begin{array}{c}2300694 \\
\text { (\% en peso) }\end{array}$ & $\begin{array}{c}2300694 \\
\text { (\% en peso) }\end{array}$ & $\begin{array}{c}2300694 \\
\text { (\% en peso) }\end{array}$ & $\begin{array}{c}2300694 \\
\text { (\% en peso) }\end{array}$ & $\begin{array}{l}2301051.5 \\
\text { (\% en peso) }\end{array}$ & $\begin{array}{l}2301092.3 \\
\text { (\% en peso) }\end{array}$ & $\begin{array}{c}2301098 \\
\text { (\% en peso) }\end{array}$ & $\begin{array}{l}2301092.3 \\
\text { (\% en peso) }\end{array}$ \\
\hline$\overline{\mathrm{SiO}_{2}}$ & 45.775 & 43.516 & 40.874 & 44.235 & 43.741 & 42.863 & 43.476 & 43.615 & 42.386 & 43.504 & 40.118 & 38.299 & 49.821 \\
\hline $\mathrm{TiO}_{2}$ & 1.899 & 1.614 & 3.726 & 3.441 & 3.485 & 3.064 & 3.256 & 3.247 & 3.343 & 2.79 & 3.006 & 2.323 & 3.422 \\
\hline $\mathrm{Al}_{2} \mathrm{O}_{3}$ & 8.942 & 7.574 & 14.711 & 13.72 & 13.679 & 12.761 & 13.158 & 13.137 & 13.47 & 15.29 & 12.332 & 9.746 & 15 \\
\hline $\mathrm{Fe}_{2} \mathrm{O}_{3}$ & 12.613 & 12.618 & 13.154 & 11.352 & 11.365 & 11.042 & 11.872 & 11.514 & 12.339 & 12.038 & 8.368 & 10.716 & 11.919 \\
\hline MnO & 0.65 & 0.56 & 0.193 & 0.136 & 0.118 & 0.17 & 0.13 & 0.125 & 0.131 & 0.64 & 0.135 & 0.228 & 0.273 \\
\hline MgO & 0.346 & 0.461 & 7.943 & 6.192 & 7.459 & 5.994 & 7.414 & 7.387 & 7.425 & 3.331 & 3.826 & 8.201 & 2.38 \\
\hline $\mathrm{CaO}$ & 13.862 & 16.512 & 5.595 & 8.152 & 8.639 & 9.127 & 10.355 & 10.719 & 9.12 & 7.548 & 13.532 & 11.858 & 4.009 \\
\hline $\mathrm{Na}_{2} \mathrm{O}$ & 0.091 & 0.014 & 0.249 & 2.139 & 1.12 & 1.599 & 0.965 & 0.852 & 0.433 & 4.198 & 1.583 & 1.108 & 2.962 \\
\hline $\mathrm{K}_{2} \mathrm{O}$ & 0.075 & 0.054 & 3.476 & 3.276 & 4.139 & 3.277 & 3.868 & 4.147 & 4.502 & 0.306 & 2.258 & 0.399 & 2.289 \\
\hline $\mathbf{P}_{2} \mathbf{O}_{5}$ & 0.272 & 0.32 & 0.946 & 0.856 & 0.82 & 0.766 & 0.808 & 0.8 & 0.788 & 0.466 & 0.766 & 0.54 & 0.819 \\
\hline PXC & 16.07 & 17.46 & 8.9 & 5.4 & 4.03 & 8.96 & 3.37 & 3.32 & 5.43 & 10.25 & 13.89 & 17.27 & 6.94 \\
\hline SUMA & 100.595 & 100.703 & 99.767 & 98.899 & 98.595 & 99.623 & 98.672 & 98.863 & 99.367 & 100.361 & 99.814 & 100.688 & 99.834 \\
\hline
\end{tabular}

$\mathrm{PXC}=$ Pérdida por calcinación; todo el Fe está expresado como $\mathrm{Fe}_{2} \mathrm{O}_{3}$

Tabla 2. Concentración de elementos traza por ICPMs (Lab. UNAM) de trece muestras de los diques de microgabro del bloque Exótico El Terrero presente dentro del Complejo El Chilar.

\begin{tabular}{|c|c|c|c|c|c|c|c|c|c|c|c|c|c|c|c|}
\hline Muestra & $\begin{array}{c}\mathbf{0 7 0 2}-\mathbf{5 A} \\
(\mathrm{ppm})\end{array}$ & $\begin{array}{c}\mathbf{0 7 0 2}-\mathbf{5 B} \\
(\mathrm{ppm})\end{array}$ & $\begin{array}{c}0702-7 \\
(\mathrm{ppm})\end{array}$ & $\begin{array}{c}\mathbf{0 7 0 2}-\mathbf{8} \\
(\mathrm{ppm})\end{array}$ & $\begin{array}{c}0702-9 \\
(\mathrm{ppm})\end{array}$ & $\begin{array}{c}0702-10 \\
(\mathrm{ppm})\end{array}$ & $\begin{array}{c}\mathbf{0 7 0 2}-11 \mathbf{A} \\
(\mathrm{ppm})\end{array}$ & $\begin{array}{c}\mathbf{0 7 0 2} \\
(\mathrm{ppm})\end{array}$ & $\begin{array}{l}-11 \mathbf{B} \\
* *(\mathrm{ppm})\end{array}$ & $\begin{array}{c}0702-11 C \\
(\mathrm{ppm})\end{array}$ & $\begin{array}{c}0702-12 \\
(\mathrm{ppm})\end{array}$ & $\begin{array}{c}0702-13 \\
(\mathrm{ppm})\end{array}$ & $\begin{array}{c}\mathbf{0 7 0} \\
(\mathrm{ppm})\end{array}$ & $\begin{array}{l}2-14 \\
* *(\mathrm{ppm})\end{array}$ & $\begin{array}{c}0702-15 \\
(\mathrm{ppm})\end{array}$ \\
\hline $\mathrm{Ba}$ & 1224 & 733.9 & 5560.9 & 5554.9 & 8403.6 & 5559.5 & 8942.1 & 9862.4 & 9290 & 7433.8 & 654.2 & 6560.3 & 291.4 & 256 & 2554.1 \\
\hline $\mathrm{Be}$ & 1.1 & 1.2 & 3 & 1.6 & 2.3 & 1.6 & 2.3 & 2.5 & - & 2.4 & 0.6 & 1.1 & 1.3 & - & 1.7 \\
\hline $\mathrm{Ce}$ & 52.4 & 42.6 & 101.8 & 98.6 & 95.2 & 88.5 & 96.2 & 103.8 & 97.8 & 99.5 & 55.9 & 88.9 & 64.3 & 58.6 & 91.6 \\
\hline Co & 88.8 & 74.3 & 62.4 & 38.1 & 63.6 & 48.5 & 55.1 & 61.8 & 53 & 43.5 & 45 & 38.9 & 91.1 & 78 & 45.1 \\
\hline${ }^{*} \mathrm{Cr}$ & 516 & 477 & 273 & 152 & 151 & 247 & 253 & 239 & - & 140 & 111 & 132 & 834 & - & 57 \\
\hline Cs & 1.9 & 2.6 & 22.4 & 43.3 & 53.5 & 21.3 & 50.2 & 60 & 46.7 & 64.6 & 6.1 & 4.4 & 7.3 & 6.4 & 13.8 \\
\hline $\mathrm{Cu}$ & 100 & 84.7 & 86.9 & 62.7 & 87.4 & 83.4 & 83.5 & 94.3 & 70 & 52.1 & 93.1 & 74.1 & 55.6 & 50 & 111.5 \\
\hline Dy & 3.2 & 3 & 6 & 6.3 & 6.4 & 5.5 & 6.3 & 6.6 & 5.5 & 6.5 & 4.5 & 5.3 & 4.4 & 3.6 & 5.3 \\
\hline Er & 1.5 & 1.3 & 2.5 & 2.6 & 2.7 & 2.3 & 2.6 & 2.8 & 2.5 & 2.7 & 1.9 & 2.2 & 1.8 & 1.7 & 2.2 \\
\hline Eu & 1.5 & 1.5 & - & - & - & - & - & - & 3.3 & - & - & - & 2.4 & 2.1 & \\
\hline Gd & 4.8 & 4 & 9.1 & 10 & 10.2 & 8.3 & 9.9 & 10.2 & 8.5 & 10 & 6.2 & 8 & 6.3 & 5.3 & 8.3 \\
\hline Hf & 3.3 & 2.4 & 6.6 & 6.3 & 6.5 & 5.6 & 5.9 & 6.7 & 6.4 & 6.7 & 2.2 & 4.7 & 4.6 & 4.5 & 5.4 \\
\hline Ho & 0.6 & 0.5 & 1 & 1.1 & 1.1 & 1 & 1.1 & 1.1 & 1 & 1.1 & 0.8 & 0.9 & 0.8 & 0.7 & 0.9 \\
\hline La & 26.1 & 21.8 & 49.6 & 47.7 & 47.5 & 44.5 & 47.9 & 51.9 & 49.6 & 46.6 & 27.4 & 44.6 & 31.5 & 28.9 & 42.9 \\
\hline $\mathbf{L i}$ & 32.3 & 20.8 & 72.5 & 34.4 & 42 & 41 & 21.9 & 23.9 & - & 50.1 & 46.7 & 25.8 & 60.5 & - & 23.5 \\
\hline Lu & 0.2 & 0.1 & 0.2 & 0.3 & 0.3 & 0.2 & 0.3 & 0.3 & 0.2 & 0.3 & 0.2 & 0.2 & 0.2 & 0.2 & 0.2 \\
\hline Mo & 6.2 & 4.8 & 0.9 & 0.2 & 0.3 & 0.7 & 0.6 & 0.7 & $<2$ & 0.3 & 0.9 & 1.3 & 1.1 & $<2$ & 3.3 \\
\hline $\mathrm{Nb}$ & 44 & 28.2 & 97.6 & 92.2 & 81.8 & 82.5 & 81 & 90.5 & 76.8 & 91.7 & 19.7 & 67.7 & 58.5 & 49.7 & 85.8 \\
\hline Nd & 28.8 & 21.8 & 55.1 & 55.6 & 55 & 49.8 & 54.4 & 58.4 & 42.9 & 56.5 & 29.3 & 49 & 36.6 & 25.9 & 49.6 \\
\hline $\mathrm{Ni}$ & 440.4 & 488.3 & 147.3 & 78.4 & 105.5 & 108 & 113 & 119.4 & 110 & 95.3 & 58.3 & 58.5 & 464.5 & 390 & 81.4 \\
\hline Pb & 1.9 & 1.6 & 6.2 & 3 & 6.2 & 3.6 & 4 & 5.4 & $<5$ & 2.1 & 2.1 & 3.5 & 2.8 & $<5$ & 3.6 \\
\hline Pr & 7 & 5.9 & 13.7 & 13.7 & 13.2 & 12.1 & 13 & 14.1 & 11.1 & 13.5 & 7.7 & 11.9 & 8.9 & 6.76 & 12.2 \\
\hline $\mathbf{R b}$ & 3.9 & 3.8 & 76 & 99.9 & 122.3 & 60.3 & 111.6 & 125.1 & 118 & 161.9 & 7.7 & 33.8 & 14.4 & 13 & 38.5 \\
\hline Sb & 10.6 & 5.7 & 1 & 1.1 & 0.8 & 0.4 & 0.9 & 1.1 & 1.2 & 0.7 & 0.1 & 0.6 & 0.7 & 1.9 & 1.7 \\
\hline Sc & 18.2 & 15.3 & 29.2 & 29.8 & 35 & 28.8 & 33.7 & 37.5 & - & 35.7 & 24.6 & 23.4 & 21.8 & & 18.6 \\
\hline Sm & 5.7 & 4.8 & 10.9 & 11.3 & 11.2 & 9.7 & 11.1 & 11.8 & 9.93 & 11.5 & 6.9 & 9.5 & 7.4 & 6.11 & 9.7 \\
\hline Sn & 1.3 & 0.9 & 2.4 & 2.2 & 2.1 & 2.1 & 2 & 2.3 & 2 & 2.4 & 1 & 2 & 1.7 & 2 & 2.2 \\
\hline $\mathrm{Sr}$ & 574 & 260.9 & 256.8 & 297.5 & 274.3 & 344.5 & 341.9 & 373.3 & 359 & 275.7 & 146.8 & 481.6 & 633.7 & 629 & 437.3 \\
\hline $\mathbf{T a}$ & 2.8 & 1.6 & 6.7 & 6.3 & 5.2 & 5.5 & 5.6 & 5.6 & 4.79 & 6.1 & 1.2 & 4 & 3.7 & 3.14 & 5.1 \\
\hline $\mathbf{T b}$ & 0.7 & 0.7 & 1.3 & 1.4 & 1.4 & 1.2 & 1.4 & 1.5 & 1.13 & 1.5 & 1 & 1.2 & 1 & 0.71 & 1.2 \\
\hline Th & 3.1 & 2.3 & 6.1 & 5.7 & 5.6 & 5 & 5.6 & 6.1 & 5.05 & 5.8 & 2.9 & 5.3 & 4 & 3.14 & 5.2 \\
\hline $\mathrm{Tm}$ & 0.2 & 0.1 & 0.3 & 0.3 & 0.3 & 0.3 & 0.3 & 0.3 & 0.315 & 0.3 & 0.2 & 0.3 & 0.2 & 0.221 & 0.3 \\
\hline $\mathbf{U}$ & 1.5 & 1.2 & 2.3 & 1.1 & 3.6 & 2.2 & 2.2 & 2.9 & 2.86 & 0.8 & 0.8 & 1.9 & 1 & 0.98 & 1.3 \\
\hline V & 225.2 & 173 & 342.7 & 312.8 & 333.1 & 292.2 & 315.7 & 356.3 & 274 & 345.6 & 201.2 & 288 & 229.7 & 190 & 340.5 \\
\hline Y & 16.5 & 15.3 & 27.8 & 30.3 & 31 & 27 & 30.3 & 31.8 & 27.2 & 31.1 & 23.3 & 26 & 21.2 & 18.5 & 25.1 \\
\hline $\mathbf{Y b}$ & 1.2 & 1 & 1.8 & 1.9 & 1.9 & 1.7 & 1.8 & 2 & 1.71 & 1.9 & 1.4 & 1.7 & 1.4 & 1.21 & 1.6 \\
\hline $\mathbf{Z n}$ & 261.3 & 219.3 & 158.3 & 152.6 & 141.3 & 135.6 & 137 & 152.1 & 140 & 151.3 & 133.1 & 88.6 & 125.1 & 120 & 177 \\
\hline $\mathrm{Zr}$ & 155 & 104.7 & 330 & 310.8 & 318.9 & 278.5 & 291.8 & 324 & 283 & 336.1 & 89.7 & 243.4 & 220.8 & 199 & 276.4 \\
\hline
\end{tabular}

Con * obtenido por FRX $\quad * *$ duplicado por ACTLABS 


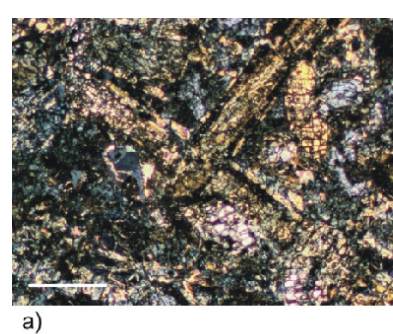

a)

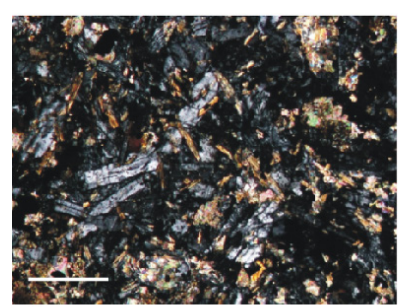

c)

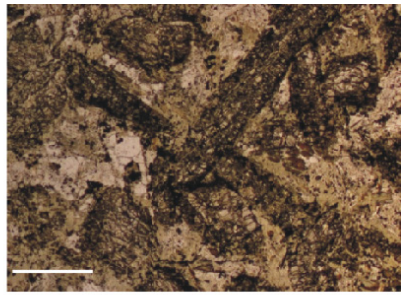

b)

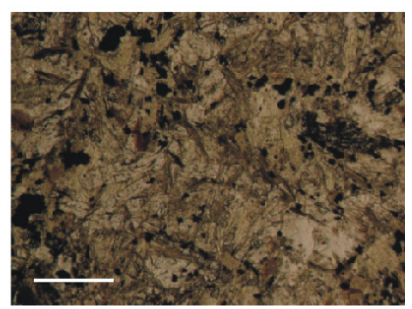

d)

Figura 3. a) Luz polarizada y b) luz natural. Textura típica de los microgabros del grupo I (muestra 9), formada por fenocristales de clinopiroxeno (augita titanífera, principalmente) entre los que existe material micro y criptocristalino (minerales arcillosos) y relictos de feldespato. c) Luz polarizada y d) luz natural. Microgabro del grupo II (muestra 14) dominado por albita con abundantes inclusiones de apatito así como mica del grupo de la biotita con orientación preferencial. Escala gráfica igual a $0.5 \mathrm{~mm}$.

microsonda del grupo I, como a continuación se describe:

Además de la titano-augita se encontraron en algunos de los cristales de piroxeno tres zonas que del interior al exterior son: 1) un núcleo de aspecto límpido y macizo (Figura 4a); 2) una zona de aspecto impuro con desarrollo de crucero, y 3 ) un borde de alteración ocasionalmente de color pardo rojizo (Figura 4b) que a veces presenta el desarrollo de crucero de anfíbol.

El análisis de microsonda electrónica permitió reconocer en dichos cristales variaciones que van de diopsida-augita hasta aegirina-augita, y en particular la ocurrencia de Fediopsida, así como de piroxeno sódico (aegirina-augita) con Mg-augita (Figura 4b, 4c). Los cristales de Fe-diopsida son bastante homogéneos en su composición desde el núcleo hasta el borde. El borde de alteración de anfíbol se puede clasificar como Ti-Fe-pargasita. Cabe mencionar que los cristales de Fe-diopsida son más magnesianos $(\mathrm{Mg} \#=0.65$ - 0.80) con respecto a los piroxenos sódicos $(\mathrm{Mg} \#<0.59)$.

Los piroxenos analizados por microsonda (WDS) e interpretados como de origen primario gracias a sus características petrográficas y texturales se pueden clasificar como Fe-diopsida cuyas concentraciones de $\mathrm{TiO}_{2}$ son 1.46 - $4.06 \%$, de $\mathrm{CaO} 22.30$ - $22.88 \%$ y de $\mathrm{Al}_{2} \mathrm{O}_{3} 3.28-8.60$ $\%$. Estos minerales recalculados en 6 oxígenos, tienen un contenido de $\mathrm{Ti}$ (total) generalmente mayor de $0.05 \%$ (0.04 - $0.12 \%$ ), Ca entre $0.89-0.92 \%$ y Al $0.14-0.38 \%$. De acuerdo a lo propuesto por Leterrier et al. (1982) estos valores son bastante más elevados a los encontrados en los clinopiroxenos típicos de magmas N-MORB acercándose a aquellos encontrados en los basaltos alcalinos. El resto de los piroxenos no se discuten aquí pues escapan a los
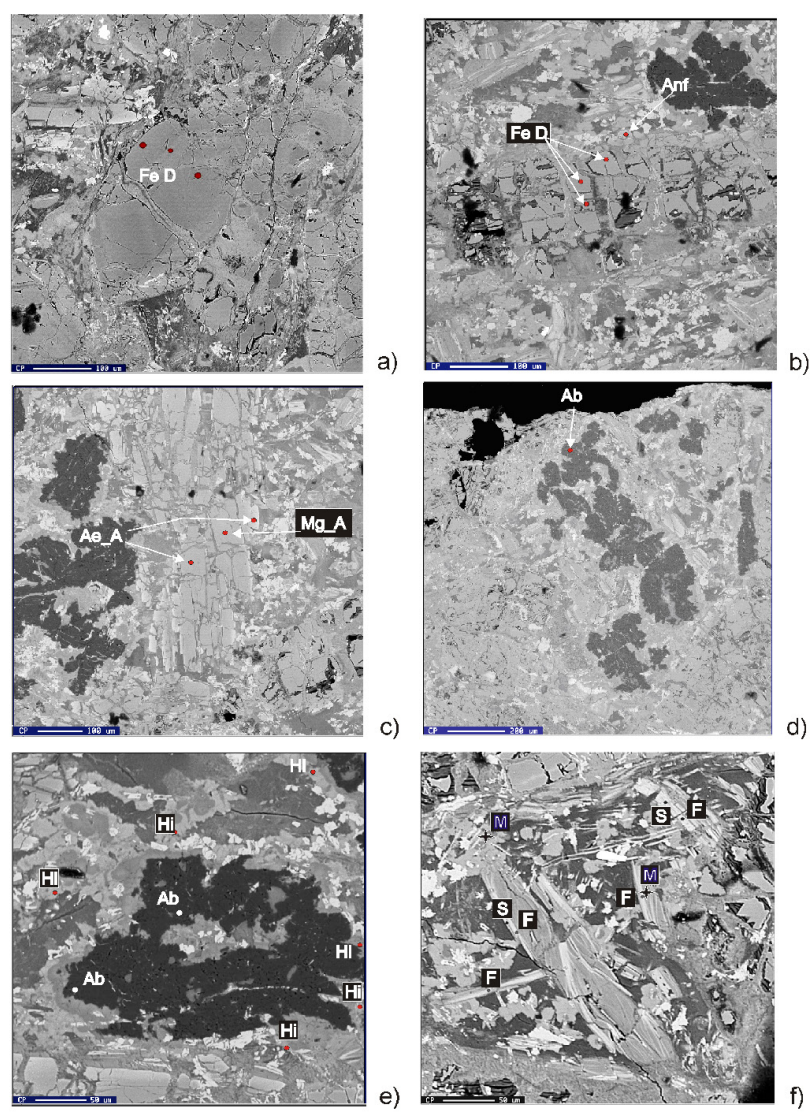

Figura 4. a) Aspecto límpido y macizo de un cristal de clinopiroxeno constituido exclusivamente por Fe-diopsida. b) Piroxeno de la muestra 9, donde el núcleo y la zona con crucero son diopsida férrica (FeD). La parte más externa del cristal (borde) está recristalizada a pargasita (Anf).c) Piroxeno con el núcleo y el borde de aegirina-augita (Ae_A), mientras que la zona con crucero corresponde a Mg-augita (Mg_A). d) Área mostrando relictos de un feldespato, donde el núcleo (tonalidad más oscura) es albita (Ab) casi pura, conservando burdamente su geometría tabular rodeada por hialofano y una matriz micro-criptocristalina. e) Detalle de un relicto de cristal de plagioclasa actualmente albita $(\mathrm{Ab})$ en tonalidad oscura, bordeada por hialofano (Hi) en tonalidades grises. f) Micas fibrosas analizadas con la microsonda, muscovita (M), flogopita férrica (F), y Mg-siderofilita (S). Nota: En todas las microfotografías de la Figura 4, los pequeños círculos indican los puntos analizados por WDS.

alcances de este artículo, pero probablemente representan procesos ocurridos posteriormente al emplazamiento de los diques de microgabro.

Los fenocristales de clinopiroxeno están rodeados por una matriz bastante desordenada y constituida aparentemente por numerosas fases cristalinas muy pequeñas. Sin embargo, las imágenes de la microsonda (electrones retrodispersados, Figura 4d) así como de catodoluminescencia (Figura 5), permitieron corroborar que originalmente también estaban presentes fenocristales de feldespatos de gran tamaño (700 $\mu \mathrm{m})$, probablemente plagioclasa. En las muestras 9, 11A, B se realizaron 24 análisis puntuales WDS de los relictos de plagioclasa: los núcleos resultaron ser de albita casi pura (Ab 98-99 mol \%) mientras que las porciones más externas están enriquecidas en $\mathrm{K}_{2} \mathrm{O}(>11 \%)$ y $\mathrm{BaO}(3.0-12.5 \%)$ 


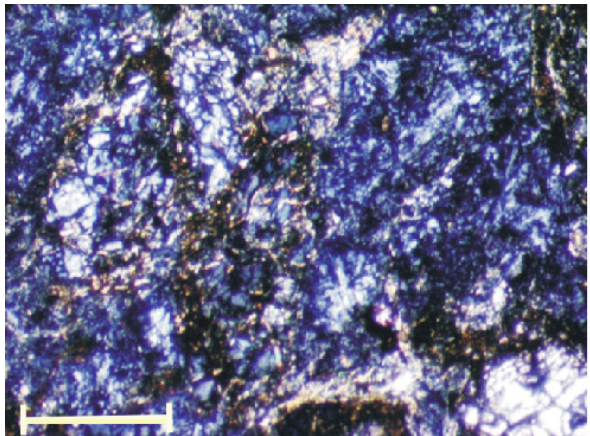

a)

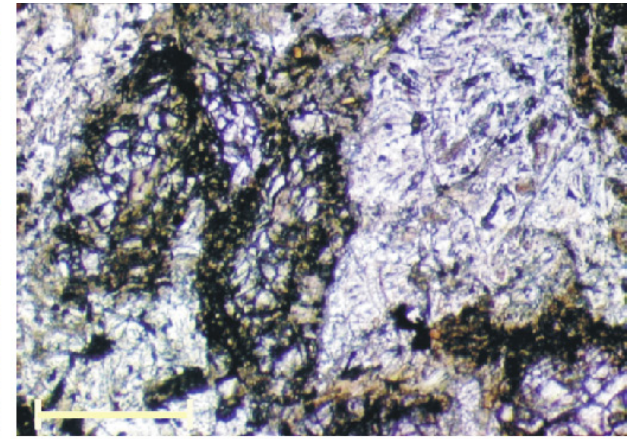

b)

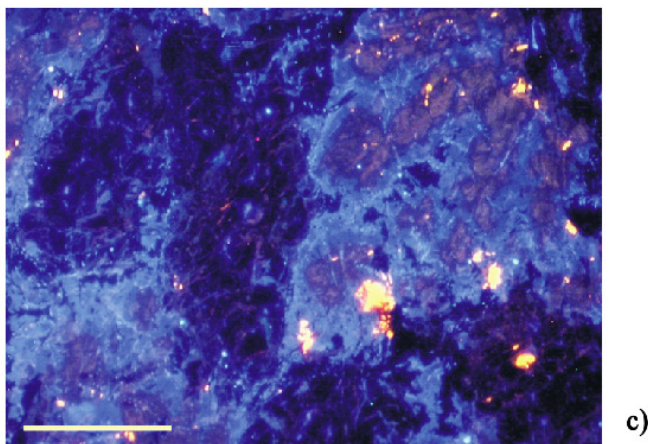

Figura 5. Fotomicrografías de la muestra 11, correspondiente al grupo I, (a) con luz polarizada, (b) luz natural, y (c) catodoluminiscencia, nótese núcleos albíticos (tonalidades naranja) con bordes de feldespato K (hialofano) azul, y algo de calcita (amarillo). Las áreas oscuras corresponden a clinopiroxenos. Escala gráfica igual a $1 \mathrm{~mm}$.

lo que permitió clasificarlos como hialofano (Figura 4e).

Por otro lado, en la matriz de las muestras menos alteradas se encuentran también calcita y micas (Figura 4f) que, según los 11 análisis WDS recalculados de acuerdo al programa Mica+ de Yavuz (2003), corresponden a Mgsiderofilitas, Fe-flogopitas y moscovitas enriquecidas en $\mathrm{BaO}$, además, óxidos de Ti y clorita. Las observaciones petrográficas y de microsonda indican ausencia de cristales de olivino o de vidrio en las muestras analizadas.

\subsubsection{Grupo II}

El grupo II tiene una mineralogía más sencilla, está compuesto por las muestras $0702-5 \mathrm{~A}$, y $5 \mathrm{~B}$ (afloramiento Chpe3), y 0702 - 12 y 0702 - 14 (afloramiento Chpe1), constituida principalmente por plagioclasa (albita) (Figuras $3 \mathrm{c}, 3 \mathrm{~d}$ ) con abundantes inclusiones aciculares de apatito. Contiene además biotita, calcita (teñida con alizarina) y otros carbonatos. Por DRX se identifico al apatito como clorapatito, a la mica como annita y a los carbonatos como dolomita o ankerita. No contiene hialofano, presenta escasos relictos de clinopiroxenos reemplazados totalmente por minerales arcillosos. Esto parece estar vinculado con la relación espacial que guardan las muestras dentro del cuerpo ígneo, ya que corresponden a los bordes de dicho cuerpo, los cuales también presentan altos valores de PxC (Figura 6A; Tabla 1).

\subsection{Geoquímica}

Todas las muestras de los grupos (petrográficos) I y II se compararon geoquímicamente, encontrándose una fuerte correlación entre ellas. Por lo que se les sigue denominando grupo I y grupo II.

En las Tablas 1 y 2 se reportan los resultados de los análisis geoquímicos de las muestras estudiadas. De acuerdo a ellos, las rocas estudiadas se caracterizan por rangos amplios en las concentraciones de casi todos los elementos mayores y traza (por ejemplo, $\mathrm{SiO}_{2}=38.3$ - 49.9 $\%, \mathrm{MgO}=0.35-8.20 \%, \mathrm{~K}_{2} \mathrm{O}=\sim 0-4.14 \%, \mathrm{Ba}=2554$ $-9862 \mathrm{ppm}, \mathrm{Ni}=58,5-158 \mathrm{ppm})$. Debido a que también los valores de pérdida por calcinación ( $\mathrm{PxC}$ ) de algunas de las muestras difieren ampliamente (3.3 - $17.5 \%$ en peso), existe la posibilidad de que parte de las variaciones en la mineralogía y la geoquímica observadas puedan atribuirse a procesos de alteración secundaria. Por lo anteriormente dicho, se seleccionaron las rocas menos alteradas, (Figura $6 \mathrm{a}, 9,11 \mathrm{~A}, 11 \mathrm{~B}$ ) y los elementos con mayor probabilidad de que sean representativos de la composición inicial para la caracterización de estas rocas.

Puesto que la mayoría de los valores originales de los elementos mayores y LILE se encuentran modificados, la discusión y conclusiones están basadas en los resultados de los elementos menos móviles. A pesar de las diferencias antes descritas, todas las muestras estudiadas pueden ser 

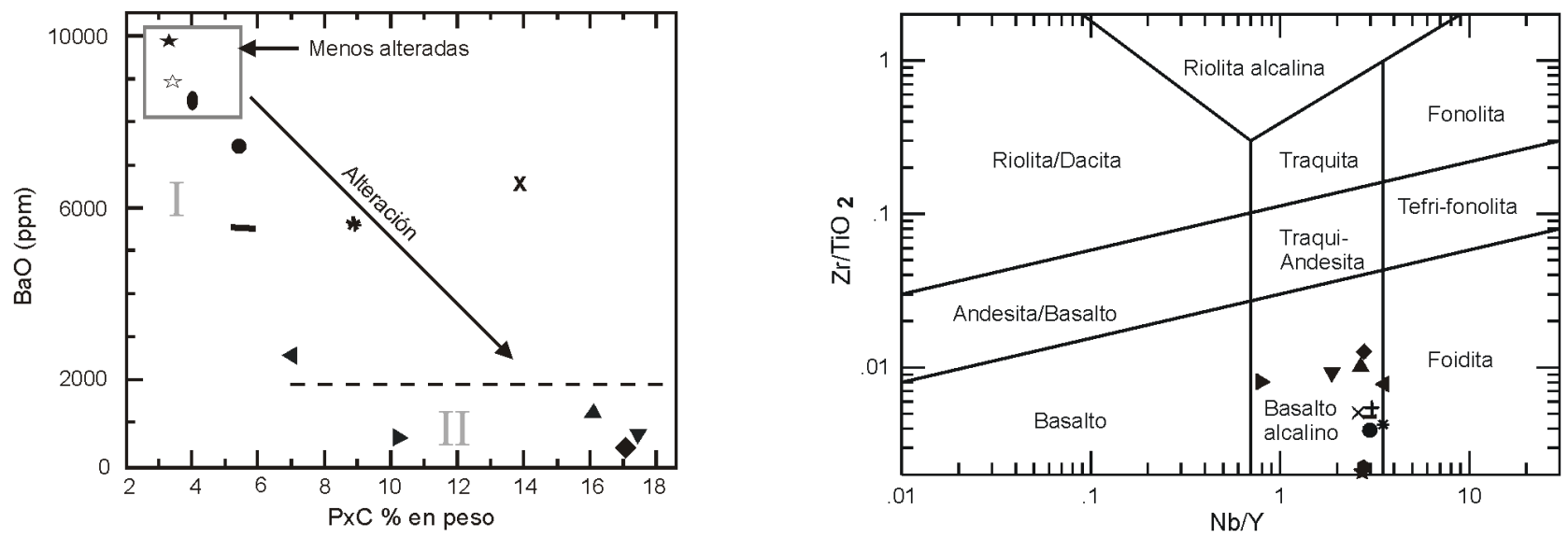

a)

b)
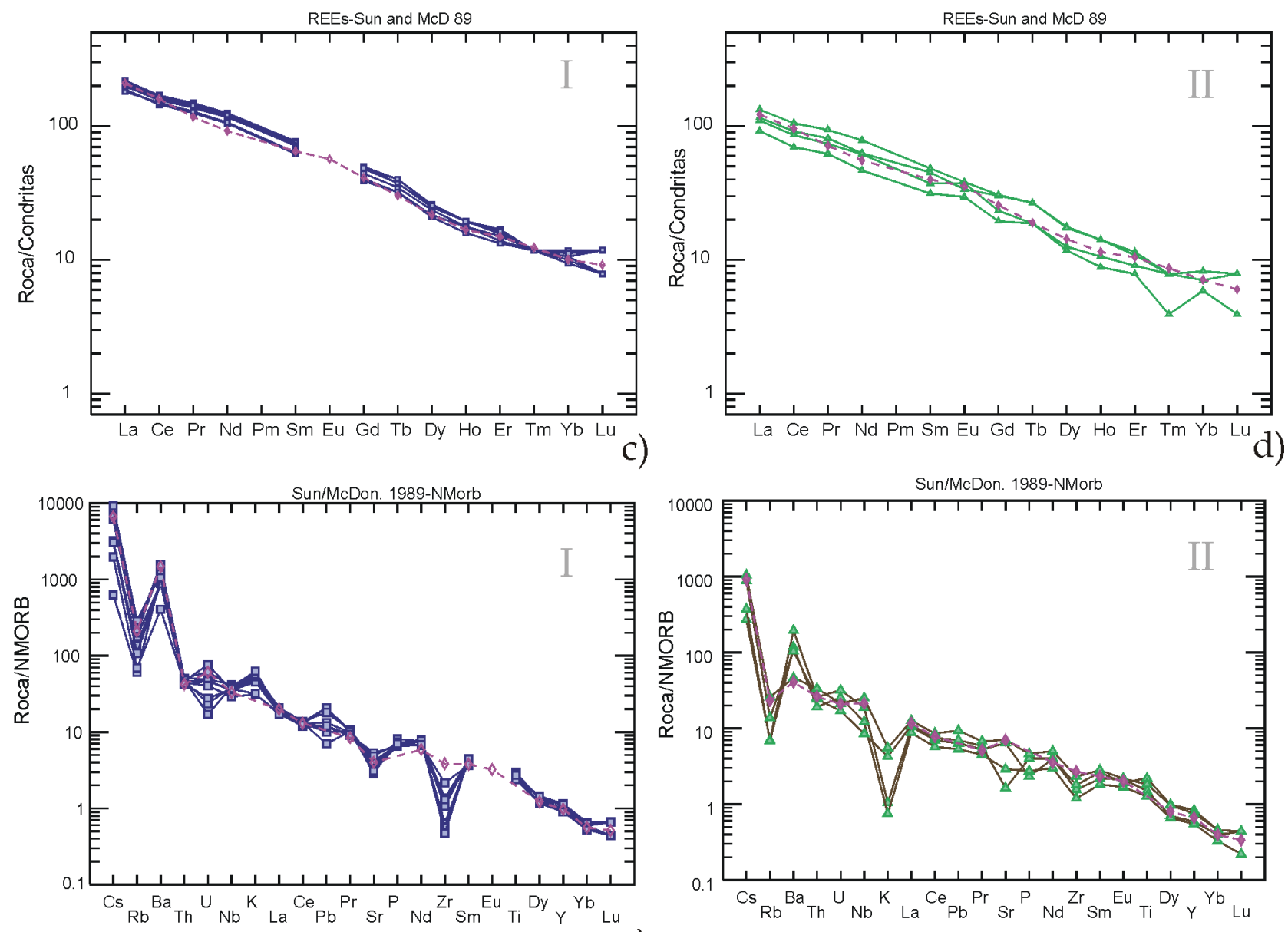

e)

Figura 6. a) Diagrama que muestra la correlación entre el índice alteración $(\mathrm{PxC}<3.3-4.0)$ con el contenido de Ba, el grupo I está formado por las muestras con menor pérdida por calcinación (PxC) y las muestras del grupo II son aquellas que presentan mayor PxC y menores concentraciones de Ba (ver Tablas 1 y 2). Las muestras menos alteradas aparecen en el cuadro superior izquierdo (11A, estrella blanca, 11B estrella negra, y 9 óvalo negro). b) Diagrama de Winchester y Floyd (1977) modificado por Pearce (1996), en donde se aprecia que las muestras estudiadas caen en el campo de los basaltos alcalinos. (c) Patrones de tierras raras de las muestras del grupo I y (d) del grupo II (valores normalizados a condrita de Sun y McDonough, 1989). La pendiente pronunciada de los patrones es típica de basaltos de intraplaca (OIB). (e) Patrones de elementos traza de las muestras del grupo I y (f) del grupo II (valores normalizados a N-MORB de Sun y McDonough, 1989). Existe un notable enriquecimiento en LILE en las muestras del grupo I, particularmente en Ba y K. 
clasificadas como basaltos alcalinos (Figura 6b) según las relaciones de elementos traza $\mathrm{Zr} / \mathrm{TiO}_{2} v s . \mathrm{Nb} / \mathrm{Y}$ (Winchester y Floyd, 1977; modificado por Pearce, 1996). Sin embargo, es de hacerse notar que las muestras del grupo I muestran un ligero incremento en el enriquecimiento en LREE (Figura 6c) con respecto a las del grupo II (Figura 6d).

La mayoría de los elementos y óxidos del grupo I presentan contenidos mayores tanto en elementos fácilmente removibles por los procesos de alteración y/o metamórficos (e.g., $\mathrm{K}_{2} \mathrm{O}, \mathrm{Ba}, \mathrm{Rb}, \mathrm{Pb}$ y U), como de elementos considerados inmóviles (e.g., $\mathrm{TiO}_{2}, \mathrm{P}_{2} \mathrm{O}_{5}, \mathrm{Nb}, \mathrm{Y}$ y REE) (Raveggi et al., 2007). Por otro lado, el grupo I se caracteriza por valores menores de PxC así como de Sr, Co, Ni, Zn y Mo.

Recalculando a 100 en base anhidra, las muestras del grupo I tienen $\mathrm{SiO}_{2}$ entre 44.9 y $53.6 \%$ en peso, con valores de álcalis $\left(\mathrm{Na}_{2} \mathrm{O}+\mathrm{K}_{2} \mathrm{O}\right)$ de 4.1 a $5.8 \%$ y relaciones $\mathrm{K}_{2} \mathrm{O} /$ $\mathrm{Na}_{2} \mathrm{O}$ casi siempre $>1$ lo que permite clasificarlas como microgabro alcalino y de afinidad potásica.

Otras características importantes de las rocas del grupo I se pueden observar en los patrones de tierras raras (Figura 6c) y de elementos incompatibles (diagrama de multielementos, Figura 6e). Con la excepción del $\mathrm{Zr}$, las rocas del Complejo El Chilar no presentan las anomalías negativas de elementos HFSE típicas de los magmas de arco (e.g., $\mathrm{Nb}$ ) o de los fundidos que han sufrido procesos de contaminación cortical (Taylor y McLennan, 1985). Algo que destaca en este grupo es un enriquecimiento inusual en los elementos más incompatibles, sobre todo Cs y Ba que están presentes en cantidades entre 3 y 4 órdenes de magnitud con respecto a un N-MORB típico. Cabe mencionar que el empobrecimiento de $\mathrm{Zr}$ podría ser el resultado de la separación de una fase mineral que concentra a este elemento (e.g., la separación de anfíbol; $\mathrm{DZr}_{\text {anfibol }}=$ 1.56; Rollinson, 1993) o bien una interferencia analítica con el Ba contenido en estas rocas. Por otro lado, las REE se caracterizan por un claro fraccionamiento entre tierras raras ligeras y pesadas $\left((\mathrm{La} / \mathrm{Yn})_{\mathrm{N}}\right.$ 17.5-20.2).

Las muestras del grupo II, tienen menores concentraciones de $\mathrm{TiO}_{2}, \mathrm{Al}_{2} \mathrm{O}_{3}$ y $\mathrm{MgO}$, y menor concentración de $\mathrm{K}_{2} \mathrm{O}$. También las concentraciones de los elementos incompatibles y REE son inferiores a las del grupo I (Figura 6d). Los patrones de tierras raras presentan una pendiente menor $(\mathrm{La} / \mathrm{Yn})_{\mathrm{N}}=13.9-16.4$.

Recalculando a 100 en base anhidra, las muestras del grupo II tienen $\mathrm{SiO}_{2}$ entre 45.9 y $54.1 \%$, con valores de álcalis $\left(\mathrm{Na}_{2} \mathrm{O}+\mathrm{K}_{2} \mathrm{O}\right)$ de 0.08 a $4.9 \%$ y relaciones $\mathrm{K}_{2} \mathrm{O} /$ $\mathrm{Na}_{2} \mathrm{O}$ regularmente $<1$, lo que permite clasificarlas como rocas basálticas o andesítico-basálticas.

Otras características importantes de las rocas del grupo II se pueden observar en los patrones de los elementos incompatibles (Figura 6f) y de las tierras raras (REE, Figura 6d). Presenta anomalías negativas de Rb, K, y una pequeña de $\mathrm{Zr}$, pero al igual que las muestras del grupo I, no presentan anomalías negativas de elementos HFSE típicas de los magmas de arco (e.g., Nb, Ta). Se observa un enriquecimiento moderado en los elementos más incompatibles, sobre todo en $\mathrm{Cs}$ y $\mathrm{Ba}$ y un ligero empobrecimiento en $\mathrm{Zr}$ con respecto a los valores de MORB.

\section{Discusión}

5.1. Ambiente tectónico de los diques que cortan al bloque exótico El Terrero, implicaciones para el Complejo El Chilar

Las relaciones de $\mathrm{Th} / \mathrm{Yb}$ vs. Ta/Yb (Pearce, 1983; Figura 7a), sugieren la presencia de una fuente de manto enriquecido tipo OIB (oceanic island basalts) para las rocas ígneas encontradas en el Complejo El Chilar. La misma afinidad se documenta con base en los diagramas de discriminación Th-Hf-Ta de Wood (1980) o V vs. Ti/1000 de Shervais (1982) (Figuras 7b y 7c). En ellos se puede observar que las rocas del grupo I y II, a pesar de las diferencias que permitieron su división, se concentran en el campo de los magmas de tipo OIB. Por otro lado, los datos obtenidos a partir del análisis por microsonda de los piroxenos, aquí considerados de origen magmático, corroboran aquello concluido con base en los análisis de roca total, mostrando que la fuente del manto de estos magmas estaba enriquecida en elementos incompatibles y con afinidad de tipo OIB (Figura 7d).

A pesar de que son pocas las muestras estudiadas, en este artículo se enfatiza el peso específico que tiene la composición geoquímica de los microgabros en la reconstrucción del ambiente tectónico del complejo El Chilar, ya que la fábrica tectónica de bloques en matriz (mélange) puede presentarse en zonas de cizalla intensa en diversos ambientes tectónicos (por ejemplo en fallas de transcurrencia importantes o cabalgaduras mayores en cinturones orogénicos), por lo que dicha fábrica, por si sola, no es suficiente para interpretar al complejo como originado por la acreción por subducción.

En cambio, si a la fábrica estructural tipo mélange se agrega la presencia de bloques de pedernal con radiolaritas, los cuales se depositan en la mayoría de los casos en el piso oceánico, esto empieza a ser una mejor evidencia de la incorporación de material derivado de una placa oceánica. $\mathrm{Si}$ a esto se suma la presencia de diques máficos que cortan exclusivamente a los pedernales, y que tiene una afinidad geoquímica de tipo isla oceánica o rift intraoceánico, se cuenta ya con dos evidencias de afinidad oceánica para éstas rocas, y que se incorporan por procesos tectónicos como bloques dentro de una matriz constituida por rocas sedimentarias de procedencia continental.

\subsection{Albitización}

Debido a que las muestras 11 y 9 son de alto $\mathrm{MgO}(>7.4$ $\%$ ), la presencia de albita casi pura es anómala y sugiere la ocurrencia de albitización temprana (Moore et al., 2006) o 


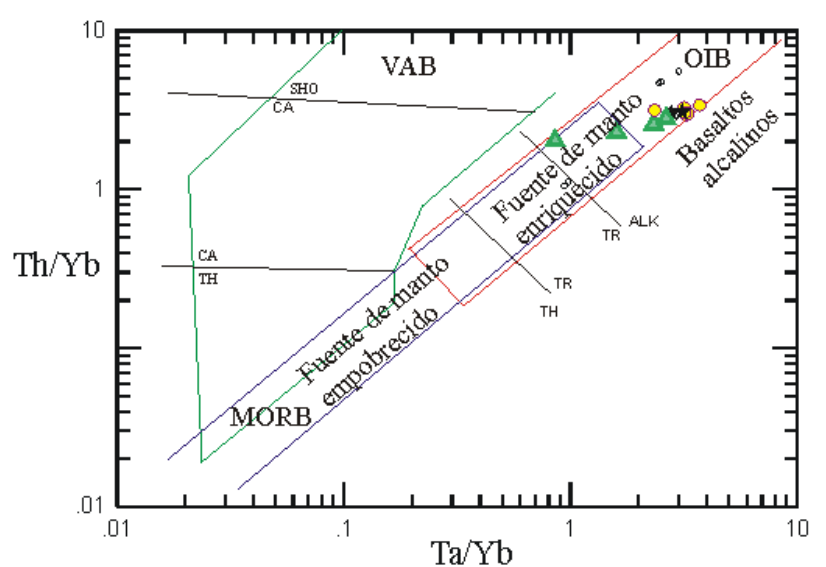

a

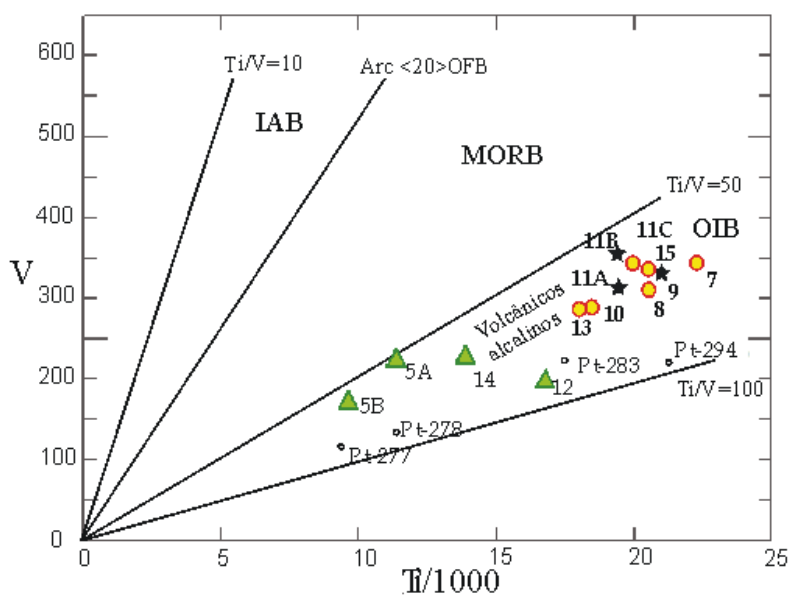

$\mathrm{C}$

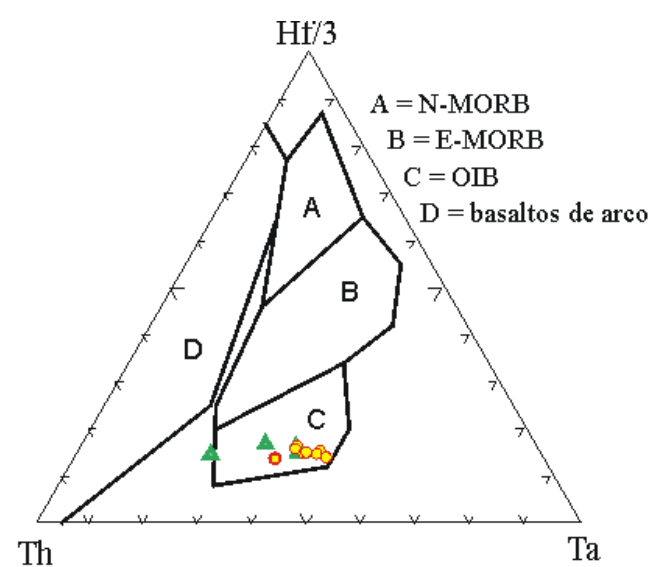

b

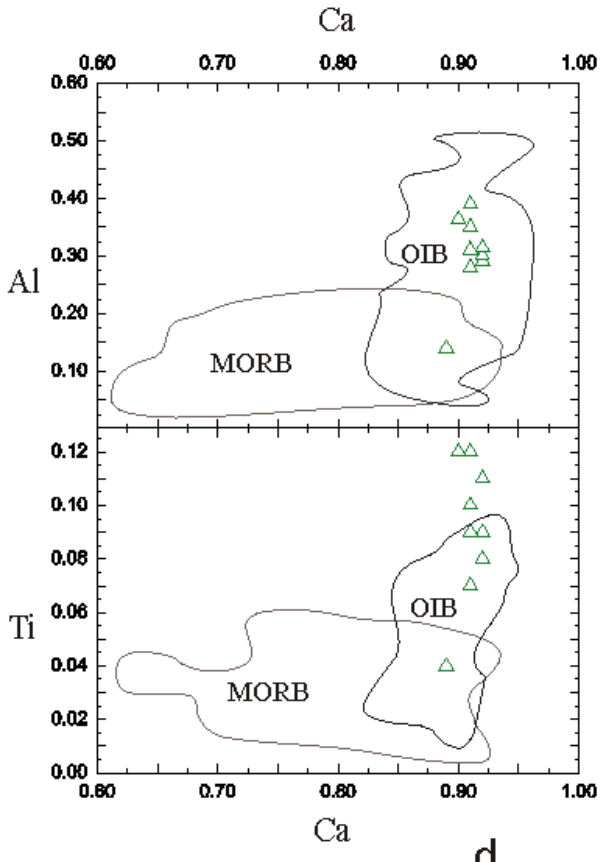

$\triangle$ Piroxenos muestra 11B

Figura 7. a) Diagrama de Th/Yb y Ta/Yb (según Pearce, 1983) en el cual las muestras de microgabros de los diques emplazados en pedernales, que forman el bloque El Terrero caen en el campo de los OIB. En el mismo diagrama se graficaron las muestras de Díaz-Salgado (2004) provenientes de bloques basálticos de la Formación Taray (círculos pequeños) y que muestran la misma afinidad de los OIB. b) Diagrama ternario Th-Ta-Hf/3 de Wood (1980) en el que se observa que todas las muestras estudiadas tienen una afinidad con los basaltos tipo OIB. c) En el diagrama V vs. Ti/1000 (Shervais, 1982) tanto las muestras analizadas en el presente estudio, como las muestras analizadas por Díaz-Salgado (2004), de la Formación Taray, caen en el campo de los OIB, sugiriendo una afinidad oceánica para los diques del bloque exótico El Terrero. d) Diagramas Al vs. Ca y Ti vs. Ca de los clinopiroxenos de las rocas magmáticas del Complejo El Chilar. También el enriquecimiento en Al y Ti sugiere una afinidad con los basaltos de tipo OIB para las muestras estudiadas. Campos tomados de Fujioka y Saito (1992).

quizás ligada a procesos inherentes a la génesis del prisma de acreción. La existencia de calcita y otros carbonatos en las muestras estudiadas es también congruente con la ocurrencia de albitización de la plagioclasa cálcia original. Asimismo, la presencia de feldespato de bario alrededor del los núcleos albíticos revela que, probablemente, el hialofano es producto de reemplazo parcial de la albita en un evento subsecuente.

\subsection{Anomalías de bario y potasio}

Las muestras con menor $\operatorname{PxC}(9,11 \mathrm{~A}, \mathrm{~B})$ exhiben un excepcional enriquecimiento en bario $(>8000 \mathrm{ppm}$, ver 
tabla 2), por lo que se hizo un estudio de microsonda para determinar las fases mineralógicas que contienen dicho elemento con el fin de comprender su génesis y relación con las rocas máficas, así como sus posibles implicaciones en la reconstrucción del ambiente tectónico. El Ba en el grupo I está concentrado principalmente en el hialofano, probablemente formado por reemplazo parcial de las plagioclasas, desarrollando zonas irregulares, heterogéneas o bordeando relictos de albita. Secundariamente se encuentra en algunas de las micas concentradas en la matriz. En el grupo II el bario está presente en concentraciones bajas (325 ppm), no determinándose qué minerales lo contienen. Estas concentraciones son bastante inferiores a las observadas en las rocas del grupo I.

Tanto las concentraciones de bario como de potasio pueden ser enriquecidas por procesos secundarios, tales como hidrotermalismo submarino o reacciones con fluidos asociados a la subducción (Blanco-Quintero et al., 2011; Blanco-Quintero et al., 2010) durante la formación del prisma de acreción o también a circulación de fluidos durante las etapas de plegamiento en ambiente cortical. Es difícil distinguir entre estos tipos de alteraciones, sin embargo algunos autores han observado que el bario generalmente no está tan enriquecido como el $\mathrm{K}, \mathrm{Rb}$ y Ce durante los procesos de alteración hidrotermal submarina (Staudigel et al., 1996; Kelley et al., 2003).

Estudios sobre la dinámica de fluidos en zonas de subducción llevados a cabo por el Ocean Drilling Program (Solomon, 2007), documentan que aproximadamente el $60 \%$ del Ba sedimentario en la margen continental de Costa Rica se pierde en la zona de subducción superficial mientras que 20 - $30 \%$ de él es subducido y se recicla al arco. Esto parece apoyar la hipótesis de que el bloque El Terrero fue parte de una zona de subducción superficial donde se enriqueció por la removilización de Ba sedimentario.

La ocurrencia de Ba sedimentario asociada a la de sílice biogénico en ambientes modernos profundos ( $>2000 \mathrm{~m})$ ha sido documentada en el mar Arábigo (Schenau et al., 2001) donde se ha concluido que la ocurrencia de dicho elemento está íntimamente ligada a la presencia de ópalo biogénico. En Costa Rica (Solomon, 2007), Perú y Japón (Torres et al., 1996) también se ha encontrado Ba asociado a secuencias profundas de la trinchera y a zonas de subducción. En el registro geológico la ocurrencia de $\mathrm{Ba}$, se tiene documentada en el noroeste de E.U.A., Nevada (Papke, 1984), y California (Sorensen et al., 1997), donde se encuentra asociada a sedimentos de aguas profundas con pedernales y antiguas zonas de subducción.

Una posibilidad más podría ser que el Ba y el K sean magmáticos, es decir ligados a una fuente del manto. No obstante, con los datos disponibles hasta la fecha esta hipótesis es la menos probable por las siguientes razones. Primero, las concentraciones de $\mathrm{Ba}$ en los diques del Complejo El Chilar ( $>8000$ ppm) son muy superiores a las encontradas en los magmas ultrapotásicos de cualquier parte del mundo; además están ausentes los minerales típicos de las rocas ultrapotásicas (e.g., leucita, sanidino, kalsilita, melilita, etc.). Finalmente, el bario se concentra en el hialofano el cual se interpreta como originado por reemplazo parcial de feldespatos preexistentes.

Por otra parte, el enriquecimiento de Ba apoya la interpretación sobre el origen del bloque exótico El Terrero como un bloque tectónico que originalmente formaba parte del fondo oceánico siendo una cobertura sedimentaria asociada a montes submarinos y que fue fragmentada e incorporada a un prisma de acreción. Sin embargo, se requiere de estudios más detallados para corroborar el origen de las anomalías de $\mathrm{Ba}$ y $\mathrm{K}$.

\subsection{Comparación con rocas similares de la Formación Taray y escudos oceánicos actuales}

En adición a lo anterior, las muestras analizadas en el presente estudio, fueron comparadas con muestras de la Formación Taray con firmas geoquímicas similares, analizadas por Díaz-Salgado, (2004) (Figuras 7a-c). Como se mencionó anteriormente, la Formación Taray ubicada en el norte del estado de Zacatecas, también contiene bloques exóticos incluidos en una matriz siliciclástica (Anderson et al., 1990, 2005; Díaz-Salgado, 2004); en particular, hay mucha similitud composicional entre un conjunto de bloques de basalto de dicha formación (bloques 3, 4, y 5; muestras PT-277, 278 283, 294 de Díaz-Salgado, 2004) y las muestras analizadas en el presente estudio (Figuras 7 - 8). La Formación Taray se ha interpretado como un complejo de subducción y presenta una posición estratigráfica, edades relativas y estilo de deformación (Centeno-García, 2005; Díaz-Salgado et al.,, 2003; Díaz-Salgado, 2004; CentenoGarcía et al., 2005; Anderson et al., 1990) muy parecidas a las del Complejo El Chilar, aunque los bloques exóticos de Taray se caracterizan por una mayor variedad composicional con respecto a los de El Chilar, debido a que también existen bloques de basalto mucho menos enriquecidos en elementos incompatibles y de afinidad N-MORB. Sin embargo, debido a que el conocimiento sobre el Complejo El Chilar es muy reciente, no se descarta la existencia de este último tipo de bloques $\mathrm{u}$ otros reportados en Taray.

Las similitudes composicionales entre los diques de microgabro de El Chilar y las rocas basálticas contenidas en algunos bloques de Taray nos lleva a proponer que los magmas que los originaron tuvieron un ambiente tectónico común y se generaron por fusión parcial de una fuente de manto enriquecida de tipo OIB. Con excepción de los LILE, patrones parecidos a las rocas del grupo I de El Chilar se observan frecuentemente en basaltos intra-oceánicos modernos (Figura 9), como por ejemplo, aquellos de la isla de Cocos en el Pacifico de Costa Rica. Dicha similitud se resalta en la figura 9, que muestra la comparación gráfica entre la composición de las muestras estudiadas en el presente trabajo y la de escudos oceánicos actuales (seamounts) en el Pacífico (Harpp et al., 2005; Hawkins et al., 1987; Janney y Baker, 1995; Eggins et al., 1991), y 


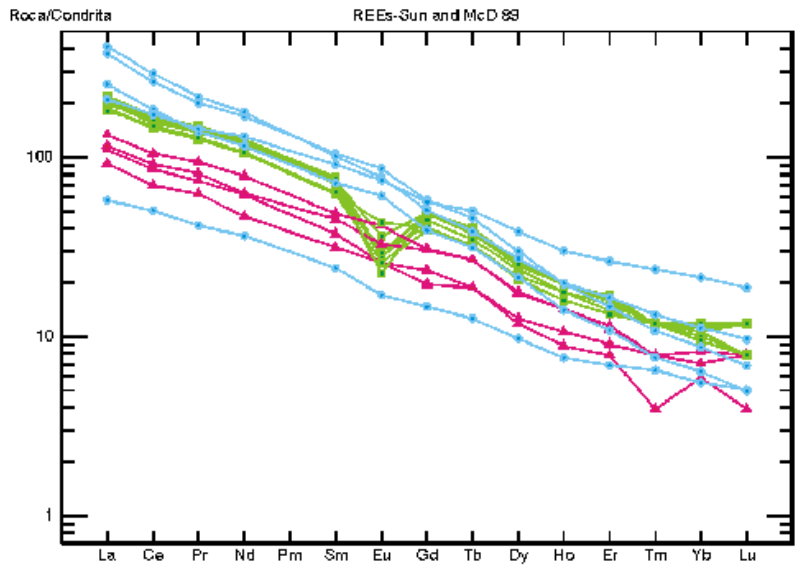

a)

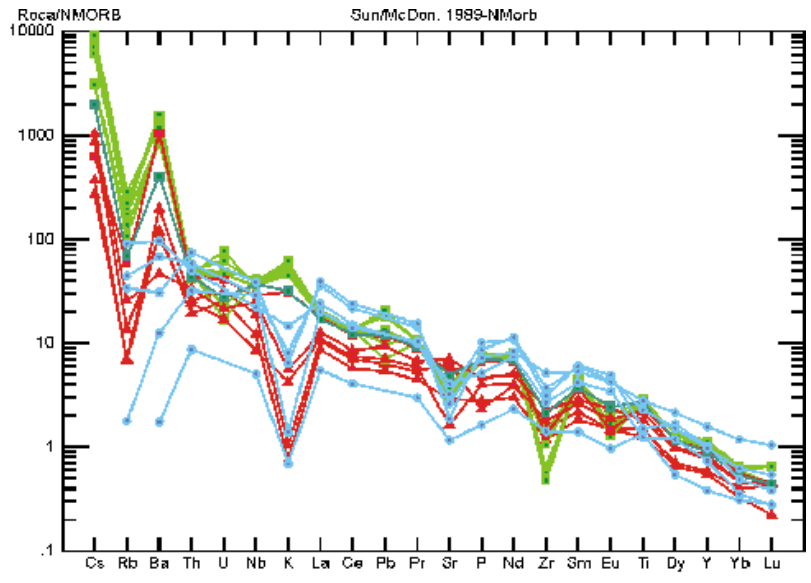

b)

Figura 8. Diagramas que comparan (a) tierras raras y (b) elementos traza de muestras obtenidas en el bloque exótico El Terrero, (grupo I en verde y grupo II en rojo), con muestras de la Formación Taray de afinidad OIB (azul). Las muestras correspondientes al grupo II muestran mayores similitudes con las muestras de la Formación Taray.

que sugiere un ambiente tectónico con condiciones análogas para el tiempo de emplazamiento de los diques.

\section{Conclusiones}

El bloque exótico El Terrero está formado por pedernales y lutitas que son cortados, previo a la deformación, por diques máficos.

Estos diques se clasifican como microgabro potásico (Le Maitre et al., 2002), y presentan variaciones mineralógicas y geoquímicas que permiten dividirlos en dos grupos (I y II).

Tanto en las muestras del grupo I como del grupo II, los elementos menos móviles presentan concentraciones similares e indican una composición de basalto alcalino con firmas de basalto de islas oceánicas, congruente con un vulcanismo desarrollado en condiciones de intra-placa.

La presencia de estos diques y su emplazamiento en pedernales con radiolaritas son interpretados aquí como la expresión del magmatismo alcalino de punto caliente que ascendió a través de fracturas en el piso oceánico, dando posiblemente origen a la existencia de montes submarinos en el entorno, mientras que los segundos se consideran parte de la cubierta sedimentaria pelágica asociada a ellos.

Posterior a su emplazamiento, los diques fueron afectados por albitización de la plagioclasa cálcica. Durante este proceso el Ca liberado constituyó la calcita y otros carbonatos reportados en el presente trabajo. Posteriormente, se deformaron junto con la roca encajonante y fueron afectados por los procesos de subducción, donde fluidos liberados a profundidades someras (Figura 10), enriquecieron de forma anómala en LILE a los microgabros $\mathrm{y}$ originaron las anomalías de $\mathrm{Ba}$ y $\mathrm{K}$ aquí documentadas.

La geoquímica de bloques con firmas afines a OIB tanto del Complejo El Chilar (aquí reportados) como de la Formación Taray es similar a la observada en escudos volcánicos (seamounts) actuales del Pacífico (Harpp et al., 2005; Hawkins et al., 1987; Janney y Baker, 1995; Eggins et al., 1991), lo que sugiere un escenario tectónico similar.

Desde el punto de vista regional, el Complejo El Chilar, conjuntamente con la Formación Taray son probablemente los remanentes de un prisma de acreción desarrollado en la margen occidental del microcontinente Oaxaquia en algún momento entre el Triásico tardío y el Jurásico temprano Medio.

\section{Agradecimientos}

Los autores agradecen a los doctores Rafael BarbozaGudiño, Uwe Martens y Dr. Antoni Camprubí, árbitros y editor respectivamente, las correcciones y sugerencias que hicieron durante la revisión del manuscrito original. También agradecemos por la realización de los análisis geoquímicos y discusión de sus resultados, al Ing. Quim. Rufino Lozano S. (Laboratorio de FRX), al Dr. Juan Pablo Bernal, (Laboratorio de ICPMS), a la M. en C. Elena Lounejeva (Laboratorio de Química Ultrapura) y a la Dra. Teresa Pi (Laboratorio de DRX) todos ellos del Instituto de Geología de la UNAM. Especiales gracias al Ing. Carlos Linares por su apoyo en la microsonda (Laboratorio Universitario de Petrología, Instituto de Geofísica), a Gerardo Zenteno por la elaboración de las figuras finales.

La presente investigación fue posible gracias al apoyo de la Universidad Nacional Autónoma de México a través de recursos asignados al proyecto PAPIIT IN115208. 

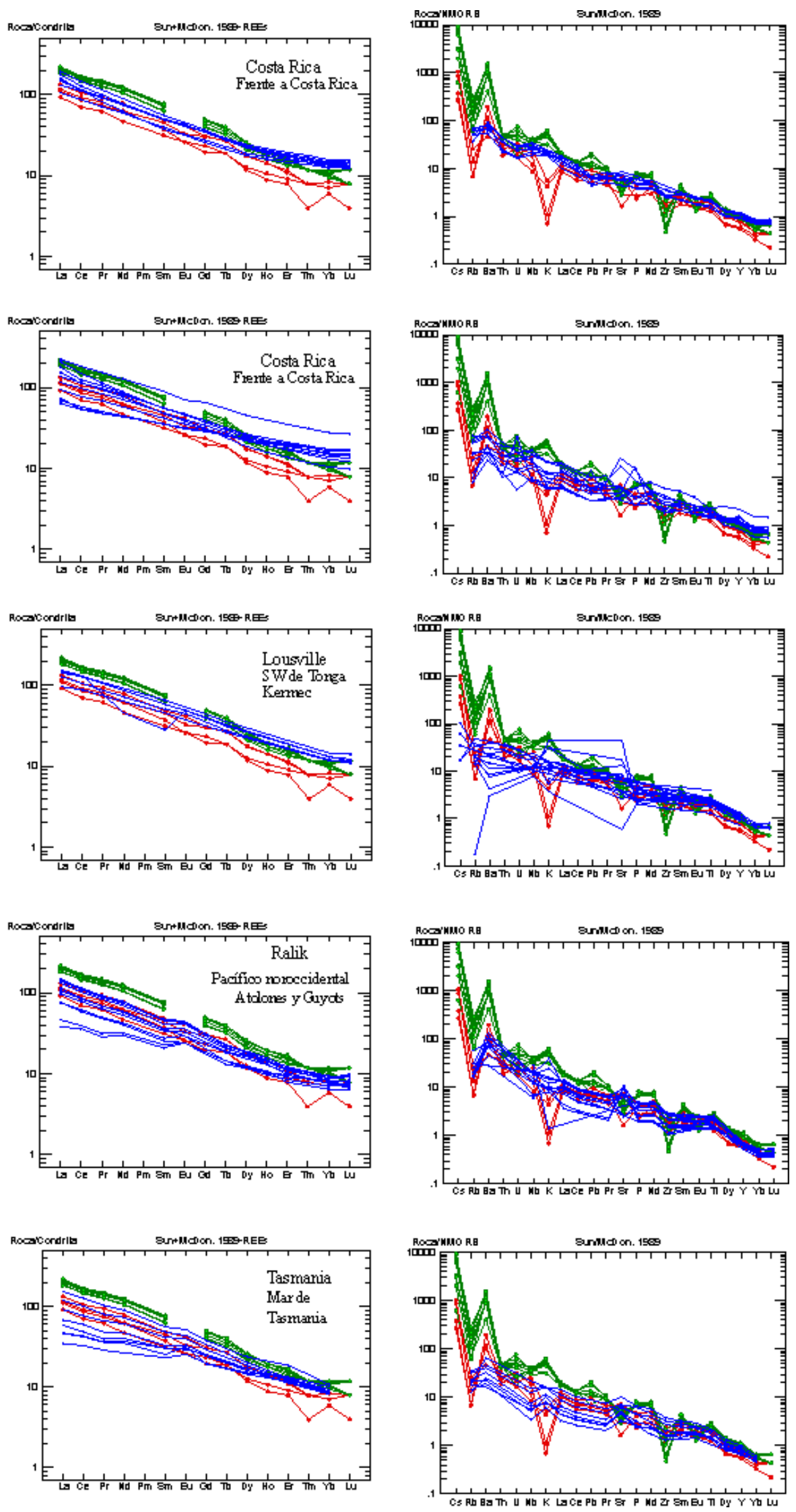

Figura 9. Diagramas que comparan tierras raras y elementos traza de muestras obtenidas en el bloque exótico El Terrero, grupo I (verde) y grupo II (rojo), con muestras provenientes de escudos oceánicos actuales (seamounts): se puede observar que los diques de microgabro del Complejo El Chilar presentan una gran similitud con los magmas que alimentan a las islas oceánicas actuales. 

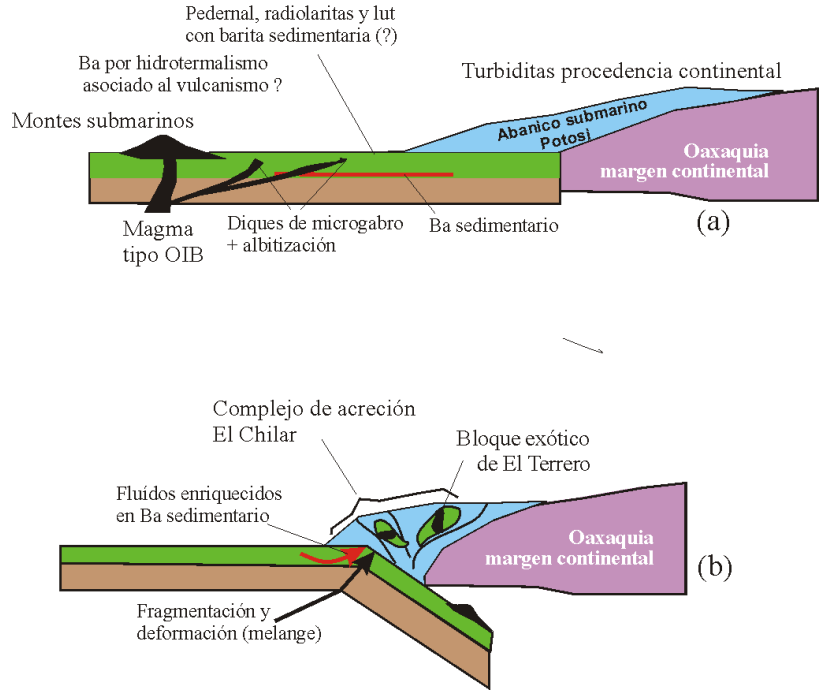

Figura 10. Modelo que muestra el origen del bloque exótico El Terrero y el Complejo El Chilar.

\section{Referencias}

Anderson, T.H., McKee J.W., Jones, N.W., 1990, Jurassic(?) Mélange in north central Mexico (resumen), Geological Society of America Abstracts with Programs, 22, 3.

Anderson T.H., Jones, N.W. McKee, J.W., 2005, The Taray Formation: Jurassic (?) mélange in northern Mexico - Tectonic implications, en Anderson, T.H., Nourse, J.A., McKee, J.W., Steiner, M.B. (eds.), The Mojave-Sonora Megashear Hypothesis: Development, Assessment, and Alternatives: EUA, Geological Society of America Special Paper 393, 427-455.

Barboza-Gudiño J.R., 2012, Sedimentary Tectonics and Stratigraphy: The Early Mesozoic Record in Central to Northeastern Mexico, en Ömer Elitok (ed.), Stratigraphic Analysis of Layered Deposits, InTech, disponible en: http://www.intechopen.com/books/stratigraphicanalysis-of-layereddeposits/sedimentary-tectonics-and-straigraphythe-early-mesozoic-record-in-central-to-northeastern-mexico, 255-278.

Barboza-Gudiño, J.R., Zavala-Monsiváis, A., Venegas-Rodríguez, G., Barajas-Nigoche, L.D., 2010, Late Triassic stratigraphy and facies from northeastern Mexico - Tectonic setting and provenance: Geosphere, 6, 621-640.

Berkland, J.O., Raymond, L.A., Kramer, J.C., Moores, E.M., O’Day, M., 1972, What is Franciscan?: American Association of Petroleum Geologists. Bulletin, 56, 2295-2302.

Blanco-Quintero, I.F., Lázaro, C., García-Casco, A., Proenza, J., RojasAgramonte, Y., 2011, Barium-rich fluids and melts in the subduction environment (La Corea and Sierra del Convento mélanges, eastern Cuba): Contributions to Mineralogy and Petrology, 162, 395-413.

Blanco-Quintero, I.F., Lázaro, C., García-Casco, A., Proenza, J., 2010, $\mathrm{Ba}$-sequestering phases in the subduction environment (eastern Cuba mélanges): implications for arc magmas: Macla, 13 49-50

Carrillo-Martínez., M., 1998 (2000), Resumen de la geología de la Hoja Zimapán, estados de Hidalgo y Querétaro, escala 1:100000: México, D.F. Universidad Nacional Autónoma de México, Instituto de Geología, Cartas Geológicas de México serie 1:100000, 1 mapa con texto.

Centeno-García, E., 2005, Review of Upper Paleozoic and Lower Mesozoic stratigraphy and depositional environments of central and west Mexico: Constraints on terrane analysis and paleogeography, en Anderson, T.H., Nourse, J.A., McKee, J.W., Steiner, M.B. (eds.), The Mojave-Sonora Megashear Hypothesis: Development, Assessment, and Alternatives: EUA, Geological Society of America Special
Paper 393, 233-258.

Centeno-García E., Gehrels, G., Díaz-Salgado C., Talavera-Mendoza, O., 2005, Zircon Provenance of Triassic (Paleozoic?) turbidites from central and western Mexico: Implications for the early evolution of the Guerrero arc (resumen), en Geological Society of America, Cordilleran Section, 101st annual meeting, San Jose, California, EUA, Geological Society of America, Abstracts with Programs, 37,64 .

Centeno-García, E., Ruiz, J., Coney, P.J., Patchett, P.J. Ortega-Gutiérrez, F., 1993. Guerrero terrane of México: its role in the Southern Cordillera from new geochemical data: Geology, 21, 419-422.

Centeno-García, E., Silva-Romo, G., 1997, Geology, geochemistry, and tectonic evolution of central Mexico during Mesozoic time: Revista Mexicana de Ciencias Geológicas, Instituto de Geología UNAM, 14, 244-260.

Dávila-Alcocer, V.M., Centeno-García. E., 2006, Stratigraphy of Toliman Region, Queretaro State, Central Mexico and its role in the evolution of the continental margin and terrane accretion (resumen), en Geological Society of America, Cordilleran Section, $102^{\text {nd }}$ annual meeting, Anchorage, Alaska, EUA, Geological Society of America, Abstracts with Programs, 38, 35.

Dávila-Alcocer, V.M., Centeno-García, E., Barboza-Gudiño, R.,Valencia, V., Fitz, D.E., 2008, Detrital Zircon Ages from the El Chilar Accretionary Complex and Volcaniclastic Rocks of the San Juan de la Rosa Formation, Toliman, Queretaro, Mexico (resumen), en Geological Society of America, Cordilleran Section, Joint meeting, Houston, Texas, EUA, Geological Society of America, Abstracts with Programs, 40, 198.

Dávila-Alcocer, V.M., Centeno-García, E., Valencia, V., Fitz D.E., 2009, Una nueva interpretación de la estratigrafía de la Región de Tolimán, Estado de Querétaro: Boletín de la Sociedad Geológica Mexicana, 61, 491-497.

Díaz-Salgado, C., 2004, Caracterización tectónica y procedencia de la Formación Taray, región de Pico de Teyra, Estado de Zacatecas: D.F., México, Universidad Nacional Autónoma de México, Tesis de maestría, $95 \mathrm{p}$.

Díaz-Salgado, C., Centeno-García, E., Gehrels, G., 2003, Stratigraphy, depositional environments, and tectonic significance of the Taray Formation, Northern Zacatecas State, Mexico (resumen), en Geological Society of America, Cordilleran Section, $9^{\text {th }}$ annual meeting, Puerto Vallarta, Jalisco, México: EUA, Geological Society of America, Abstracts with Programs, 35, 71.

Eggins, S.M., Green, D.H., Falloon, T.J., 1991, The tasmantid seamounts: shallow melting and contamination of an EM1 mantle plume: Earth and Planetary Science Letters, 107, 448-462.

Festa, A., Pini, G.A., Dilek, Y., Codegone, J., 2010, Mélanges and mélange-forming processes: a historical overview and new concepts: International Geology Review, 52, 1040-1105.

Fujioka, K. Saito, S., 1992, Composition of heavy minerals from sand and sandstones of the Izu-Bonin Arc, Leg 126, en Proccedings of the Ocean Drilling Program, Scientific Results, 126, 155-169.

Harpp, K.S., Wanless, R.K., Otto, R.H., Hoernle, K.A., Werner, R., 2005, The Cocos and Carnegie Aseismic Ridges: A Trace Element Record of Long-Term Plume-Spreading Center Interaction: Journal Petrology 46, 109-133.

Hawkins, J.W. Jr., Lonsdale, P., Batiza, R., 1987, Petrologic Evolution of The Louisville Seamount Chain, en Keating, B., Fryer, P., Batiza, R. (eds.), Seamounts, Islands, and Atolls: Washington D.C., American Geophysical Union, 235-254.

Janney, P.E., Baker, P.E., 1995, Petrology and geochemistry of basaltic clasts and hyaloclastites from volcanoclastic sediments at site 869: Proceedings of the Ocean Drilling Program, Scientific Results, 143, 263-276.

Kelley, K.A., Plank, T. Ludden J. Staudigel, H., 2003, Composition of altered oceanic crust at ODP Sites 801 and 1149: Geochemistry Geophysics Geosystems, 4, 8910.

Kusky, T.M., Bradley D.C., 1999, Kinematic analysis of mélange fabrics: examples and applications from McHugh Complex, Kenai Peninsula, Alaska: Journal of Structural Geology, 21, 1773-1796. 
Le Maitre, R.W. A. Streckeisen, B. Zanettin, M.J. Le Bas, B. Bonin, P Bateman, G. Bellieni, A. Dudek, S. Efremova, J. Keller, J. Lamere, P.A. Sabine, R. Schmid, H. Sorensen, A.R. Woolley, 2002, Igneous Rocks: A Classification and Glossary of Terms, Recommendations of the International Union of Geological Sciences, Subcommission of the Systematics of Igneous Rocks, Cambridge, U.K., Cambridge University Press, $232 \mathrm{p}$.

Leterrier, J., Maury, R.C, Thonon, P., Girard, D., Marchal, M., 1982, Clinopyroxene composition as a method of identification of the magmatic affinities of paleo-volcanic series. Earth and Planetary Science Letters, 59,139-154.

López-Ramos E., 1985, Geología de México (3 ${ }^{\mathrm{a}}$ ed.), tomo II: México D.F. edición particular, 402 p.

Medley, E.W., 2002, Estimating block size distributions of mélanges and similar block-in-matrix rocks (bimrocks): en Hammah, R., Bawden, W., Curran, J., Telesnicki, M. (eds.), Proceedings of the $5^{\text {th }}$ North American Rock Mechanics Symposium (NARMS): Toronto Canada, 599-606.

Moore, J.C., Rowe, C., Meneghini, F., 2006, How accretionary prisms elucidate seismogenesis in subduction zones, en Moore, J.C., Dixon, T.H. (eds.), Interplate Subduction Zone Seismogenesis: New York, Columbia University Press, 288-315.

North American Commission on Stratigraphic Nomenclature (NACSN), 2005, North American stratigraphic code: American Association of Petroleum Geologists Bulletin, 89, 1547-1591.

Papke, K.G., 1984, Barite in Nevada: Nevada Bureau of Mines and Geology Bulletin 98, 125p.

Pearce, J.A., 1983, Role of the sub-continental lithosphere in magma genesis at active continental margins, en Hawkesworth, C.J., Norry, M. J. (eds.), Continental basalts and mantle xenoliths: Nantwich, UK, Shiva, 230-249.

Pearce, J.A. 1996, A user's guide to basalt discrimination diagrams, en Wyman, D. A. (ed.) Trace Element Geochemistry of Volcanic Rocks: Applications for Massive Sulphide Exploration: Canada, Geological Association of Canada, Short Course Notes 12, 79-113.

Raveggi, M., Giles, D., Foden, J., Raetz, M., 2007, High Fe-Ti mafic magmatism and tectonic setting of the Paleoproterozoic Broken Hill Block, N.S.W. Australia: Precambrian Research, 156, 55-84.

Raymond, L.A., 1984, Classification of melanges, en Raymond, L.A. (ed.), Mélanges: Their nature, origin, and significance: Geological Society of America Special Paper 198, 7-20.

Rollinson, H.R., 1993, Using Geochemical Data: Evaluation, Presentation, Interpretation: England, Longman Scientific \& Technical, 352 p.

Schenau, S.J., Prins, M.A., De Lange, G.J., Monnin, C., 2001, Barium accumulation in the Arabian Sea: controls on barite preservation in marine sediments: Geochimica et Cosmochimica Acta, 65, $1545-1556$
Shervais, J.W., 1982, Ti-V plots and the petrogenesis of modern and ophiolitic lavas: Earth and Planetary Science Letters, 59, 101-118.

Silva-Romo, G., Arellano Gil, J., Mendoza Rosales, C., Nieto Obregon, J., 2000, A submarine fan in the Mesa Central, Mexico: Journal of South American Earth Sciences, 13, 429-442.

Solomon, E. A., 2007, The dynamics of fluid flow and associated chemical fluxes at active continental margins: San Diego, U.S.A., University of California, Tesis doctoral, $251 \mathrm{p}$.

Sorensen S.S., Grossman J.N., Perfit M.R., 1997, Phengite-hosted LILEenrichment in eclogite and related rocks: implications for fluidmediated mass transfer in subduction zones and arc magma genesis: Journal Petrology, 38, 3-34.

Staudigel, H., Plank, T., White, W., Schmincke, H.U., 1996, Geochemical fluxes during seafloor alteration of the basaltic upper oceanic crust: DSDP Sites 417 and 418, en Bebout, G.E., Scholl, D.W., Kirby S.H., Platt J.P. (eds.), Subduction Top to Bottom: Washington, D.C., American Geophysical Union Monograph, Series 96, 19-38.

Sun, S.S. McDonough, W.F., 1989, Chemical and isotopic systematics of oceanic basalts: implications for mantle composition and processes, en Saunders A.D., Norry M.J. (eds.), Magmatism in ocean basins: London, The Geological Society, Special Publication 42, 313-345.

Taylor, S.R., McLennan, S.M., 1985. The continental crust: its composition and evolution, Blackwell, Oxford: $321 \mathrm{p}$.

Torres, M.E., Brumsack, H.J., Bohrman, G., Emeis, K.C., 1996, Barite front in continental margin sediments: a new look at barium remobilization in the zone of sulfate reduction and formation of heavy barites in diagenetic fronts: Chemical Geology, 127, 125-139.

Winchester, J.A., Floyd P.A., 1977, Geochemical discrimination of different magma series and their differentiation products using immobile elements: Chemical Geology, 20, 325-243.

Wood, D.A., 1980, The application of Th-Hf-Ta diagram to problems of tectonomagmatic classification and to establishing the nature of crustal contamination of basaltic lavas of the British Tertiary volcanic province: Earth and Planetary Science Letters, 50, 11-30.

Yavuz, F., 2003, Evaluating micas in petrologic and metallogenic aspect: I - Definitions and Structure of The Computer Program Mica+: Computers \& Geosciences, 29, 1203-1213.

Manuscrito recibido: Agosto 8, 2012.

Manuscrito corregido recibido: Noviembre 28, 2012.

Manuscrito aceptado: Noviembre 30, 2012. 